
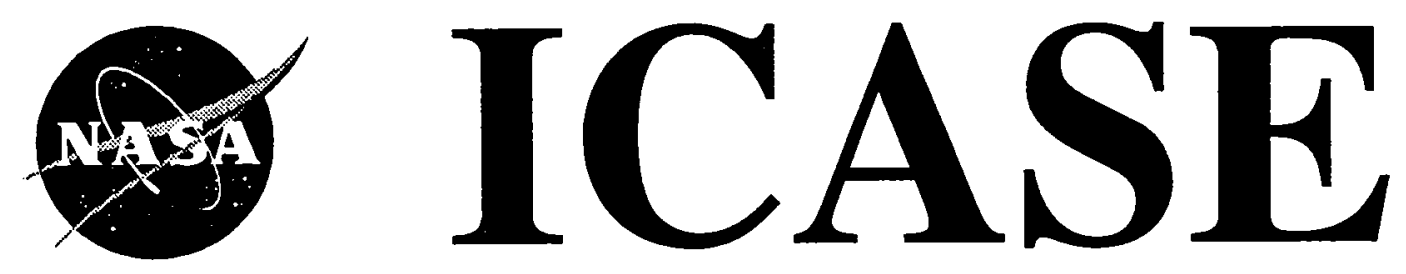

\title{
EXPERIMENTAL CONFIRMATION OF A PDE-BASED APPROACH TO DESIGN OF FEEDBACK CONTROLS
}

\section{H. T. Banks}

Ralph C. Smith

D. E. Brown

R. J. Silcox

Vern L. Metcalf

Contract No. NAS1-19480

May 1995

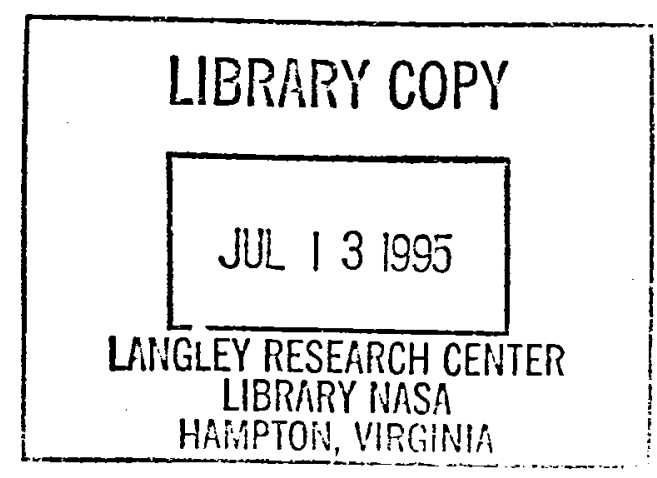

Institute for Computer Applications in Science and Engineering NASA Langley Research Center

Hampton, VA 23681-0001

SRA Operated by Universities Space Research Association 


\title{
EXPERIMENTAL CONFIRMATION OF A PDE-BASED APPROACH TO DESIGN OF FEEDBACK CONTROLS ${ }^{1}$
}

\author{
H.T. Banks \\ Center for Research in Scientific Computation \\ North Carolina State University \\ Raleigh, NC. 27695 \\ D.E. Brown \\ Lockheed Engineering and Sciences Company \\ NASA Langley Research Center \\ Hampton, VA 23681
}

\author{
Ralph C. Smith \\ Department of Mathematics \\ Iowa State University \\ Ames, IA 50011 \\ R.J. Silcox \\ Acoustics Division \\ NASA Langley Research Center \\ Hampton, VA 23681
}

\author{
Vern L. Metcalf \\ U.S. Army Research Laboratory \\ NASA Langley Research Center \\ Hampton, VA 23681
}

\begin{abstract}
Issues regarding the experimental implementation of PDE-based controllers are discussed in this work. While the motivating application involves the reduction of vibration levels for a circular plate through excitation of surface-mounted piezoceramic patches, the general techniques described here will extend to a variety of applications. The initial step is the development of a PDE model which accurately captures the physics of the underlying process. This model is then discretized to yield a vector-valued initial value problem. Optimal control theory is used to determine continuous-time voltages to the patches, and the approximations needed to facilitate discrete time implementation are addressed. Finally, experimental results demonstrating the control of both transient and steady state vibrations through these techniques are presented.
\end{abstract}

\footnotetext{
${ }^{1}$ The research of II.T.B. was supported in part by the Air Force Office of Scientific Research under grant. AFOSR-F49620-93-1-0198. This research was also supported by the National Aeronautics and Space Administration under NASA Contract Number NAS1-19480 while II.T.B and R.C.S. were visiting scientists at the Institute for Computer Applications in Science and Engineering (ICASE), NASA Langley Research Center, Hampton, VA 23681. Additional support was also provided in part under NASA grant NAG-1-1600.
} 


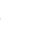




\section{Introduction}

An increasingly popular method for controlling structural vibrations is through the use of piezoceramic patches bonded to or imbedded in the structure. These patches exhibit the piezoelectric property that inplane strains are generated in response to an applied voltage. Depending upon the geometry of patch placement, location with respect to the structure's neutral surface, and the method of excitation, this provides a mechanism for generating both inplane forces and/or bending moments in the underlying structure.

The advantage of using such patches as actuators in many applications is due to the fact that they are lightweight, space efficient, relatively inexpensive, and provide a means of obtaining structural control without significantly changing the passive structural dynamics (they do not mass load the structure in the manner of a shaker or proof mass actuator). Due to their ceramic nature, they can also be molded in a variety of shapes so as to fit the structure under consideration. Moreover, rigid body torques and spillover effects are minimized due to the fact that they are fully self contained and distributed in nature. Finally, they also exhibit the inverse piezoelectric property and hence generate a voltage in response to strains in the material. Hence a single patch or patch pair can be used for either sensing or actuation. This contributes to their efficiency and application in "smart material" structures.

In addition to their utility in purely structural applications, they are also finding increasing use in structural acoustic and fluid/structure applications. Again, their advantage lies in the fact that they provide an efficient means of controlling the structure without significantly altering its passive dynamics. Through consideration of the coupling between the structure and the adjacent media, this provides a means of controlling acoustic sound pressure levels or adjacent flow dynamics.

A great deal of research in the last several years has been directed toward questions regarding the modeling of piezoceramic patch interactions with underlying structures (see [11] and the references therein), strategies for determination of optimal patch location and number, and control techniques which utilize the patches as sensors and actuators. Due to the steady state periodic nature of the dynamics in many structural and structural acoustic systems, a large number of the current control methods are based upon frequency response input/output analysis. For example, Fuller et al. have employed a feedforward filtered $\mathrm{X}$ version of an adaptive LMS algorithm to control a simply supported plate using piezoceramic actuators [18]. While such techniques have proven quite successful for controlling steady state vibrations, they do not have the capability for direct control of transient responses. Other successfully implemented methods employing piezoelectric actuators to actively control structural vibrations include feedthrough techniques [17] and velocity feedback techniques [1, 24]. In general, these methods are based upon modal techniques and are designed to control purely steady state responses. An exception to this are the experimental results reported in [24] in which transient plate vibrations, generated by an impact hammer, were reduced using an analog velocity feedback circuit.

Similar studies have demonstrated the experimental success of using surface-mounted piezoceramic patches to reduce structure-borne noise in structural acoustic systems [19, 20]. The emphasis in these studies was again on using frequency input/output analysis to control steady state dynamics. 
An alternative approach to controlling structural vibrations and sound pressure levels in structural acoustic systems is through the use of PDE-based feedback control methods. Analysis and numerical studies demonstrating these techniques for structural applications can be found in $[6,7,8,12]$ with corresponding results for structural acoustic systems given in $[4,5,10]$. These techniques start with an infinite dimensional PDE model for the system under consideration. When developing such models, care should be taken to incorporate not only the contributions due to the piezoceramic patches but also dynamics due to inexact boundary conditions [13], coupling with adjacent acoustic fields [5], as well as any other physical phenomena which affect the dynamics of the structure. In this setting, mathematical issues such as model well-posedness and approximation issues concerning simulations, parameter estimation and control can be addressed.

By approaching the problems in this manner, one can avoid the difficulties caused in purely modal methods by patch contributions, coupling between components, and inexact boundary conditions. Moreover, by combining the PDE model with appropriate time-dependent feedback control theory, one obtains a method which is equally applicable for controlling transient or steady state vibrations.

In this paper, the experimental implementation of such a PDE-based control methor is considered. While the motivating application involves the control of vibration levels for a circular plate through the excitation of surface-mounted piezoceramic patches, the general techniques described here will extend to a variety of applications. Following a brief discussion regarding the model and a Fourier/Galerkin scheme used to discretize it, relevant feedback control theory is discussed. In the discussion of the continuous and discrete time control results, two cases are considered; namely, the control of plate vibrations in the absence of a primary input force and the control of a plate driven by a periodic exogenous force. Implementation issues such as the effects of phase shifts and delays due to hardware are discussed and the experimental setup is briefly described. Finally, experimental results demonstrating the transient and steady state control results are presented. These demonstrate the effectiveness of the PDE-based controller for this system and indicate the potential of these control techniques for reducing transient and steady state dynamics in other structural and structural acoustic systems.

Finally, we offer comments on the nature of our contributions here to the literature. This paper does not contain any new theoretical results; it reports on our successful use of PDEbased methods in experiments at NASA Langley Research Center. The methods (which are theoretically sound) for parameter estimation and feedback control are based on approximation theory developed (by us and many others in the PDE control community) during the past several decades. All necessary theorems on convergence of finite dimensional parameter estimates, gains, filters, controls, observers, tracking variables, et cetera, needed for the examples treated here either have appeared or will soon appear in the research literature. Many of these theoretical results on PDE-based control have been largely viewed (especially in the engineering community) as non-implementable and, hence, as somewhat irrelevant to applied scientists and engineers. The present manuscript refutes this notion and validates the practicality and importance of much of the theoretical efforts over the past years on PDE-based control. Our group is the first, to our knowledge, to provide irrefutable evidence that one can start from basic physical laws, derive careful infinite dimensional distributed parameter or PDE control models, and successfully implement PDE control methods (with the approximations necessary to obtain finite dimensional controls) which behave as theory and simulations predict. 


\section{Circular Plate Model}

The structure under consideration is a thin circular plate with $s$ sets of piezoceramic patches bonded to the plate either singly or in pairs as depicted in Figure 2.1. Throughout this discussion, the radius and thickness of the plate are denoted by $a$ and $h$, respectively. Moreover, the density, Young's modulus, Poisson ratio, Kelvin-Voigt and air clamping parameters are given by $\rho, E, \nu, c_{D}$ and $\gamma$ while $w$ and $\tilde{g}$ are used to denote the transverse displacement and external force on the plate. Finally, the region occupied by the unstrained neutral surface of the plate is indicated by $\Gamma_{0}$.

Equations of motion for the plate can be determined from both Newtonian (force and moment balancing) and Hamiltonian (energy formulation) principles and we summarize both approaches here. The presentation will be for a general Kirchhoff plate with potentially nonaxisymmetric responses.
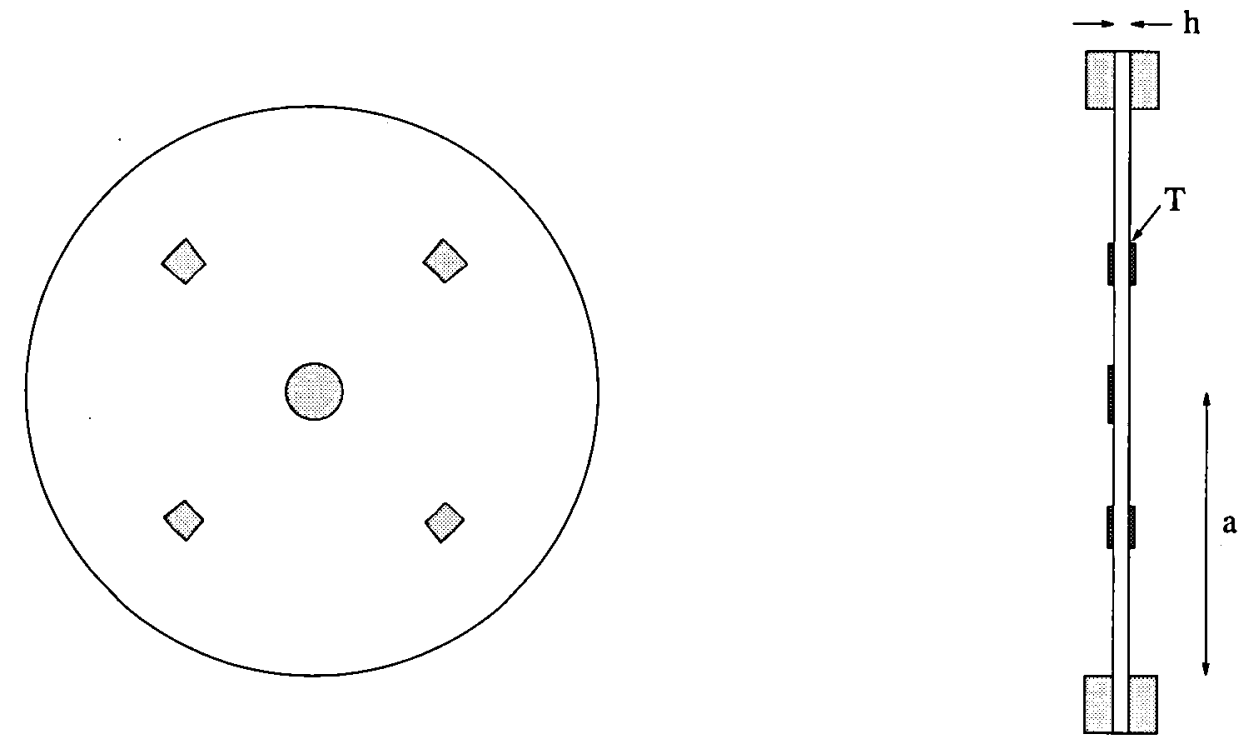

Figure 2.1. Thin circular plate with surface-mounted piezoceramic patches.

\subsection{Strong Form of Plate Model}

Considering first the model which derives from Newtonian principles, we let $M_{r}, M_{\theta}, M_{r \theta}$ denote internal moments and $\left(M_{r}\right)_{p \epsilon},\left(M_{\theta}\right)_{p \epsilon}$ denote external moments generated by the piezoceramic patches. As detailed in $[9,29]$, for a structure with patch pairs activated out-of-phase, the 
internal moments are given by

$$
\begin{aligned}
& M_{r}=-D\left(\frac{\partial^{2} w}{\partial r^{2}}+\frac{\nu}{r} \frac{\partial w}{\partial r}+\frac{\nu}{r^{2}} \frac{\partial^{2} w}{\partial \theta^{2}}\right)-c_{D}\left(\frac{\partial^{3} w}{\partial r^{2} \partial t}+\frac{\nu}{r} \frac{\partial^{2} w}{\partial r \partial t}+\frac{\nu}{r^{2}} \frac{\partial^{3} w}{\partial \theta^{2} \partial t}\right) \\
& M_{\theta}=-D\left(\frac{1}{r} \frac{\partial w}{\partial r}+\frac{1}{r^{2}} \frac{\partial^{2} w}{\partial \theta^{2}}+\nu \frac{\partial^{2} w}{\partial r^{2}}\right)-c_{D}\left(\frac{1}{r} \frac{\partial^{2} w}{\partial r \partial t}+\frac{1}{r^{2}} \frac{\partial^{3} w}{\partial \theta^{2} \partial t}+\nu \frac{\partial^{3} w}{\partial r^{2} \partial t}\right) \\
& M_{r \theta}=-D(1-\nu)\left(\frac{1}{r} \frac{\partial^{2} w}{\partial r \partial \theta}-\frac{1}{r^{2}} \frac{\partial w}{\partial \theta}\right)-c_{D}(1-\nu)\left(\frac{1}{r} \frac{\partial^{3} w}{\partial r \partial \theta \partial t}-\frac{1}{r^{2}} \frac{\partial^{2} w}{\partial \theta \partial t}\right)
\end{aligned}
$$

where $D=\frac{E h^{3}}{12\left(1-\nu^{2}\right)}$ denotes the plate stiffness. The external moments generated by the $s$ patch pairs have the form

$$
\left(M_{r}\right)_{p \epsilon}=\left(M_{\theta}\right)_{p \epsilon}=-\sum_{i=1}^{s} \mathcal{K}_{i}^{B} u_{i}(t)_{i}(r, \theta) \quad, \quad \chi_{i}(r, \theta)= \begin{cases}1 & ,(r, \theta) \in i^{t h} \text { patch region } \\ 0, & \text { elsewhere }\end{cases}
$$

where $u_{i}(t)$ is the voltage into the $i^{\text {th }}$ patch and $\mathcal{K}_{i}^{B}$ is a parameter which depends on the geometry, piezoceramic material properties and piezoelectric strain constants (see [11] for details). The internal and external moments can then be combined to yield the general plate moments

$$
\begin{aligned}
\mathcal{M}_{r} & =M_{r}-\left(M_{r}\right)_{p \epsilon} \\
\mathcal{M}_{\theta} & =M_{\theta}-\left(M_{\theta}\right)_{p \epsilon} \\
\mathcal{M}_{r \theta} & =M_{r \theta} .
\end{aligned}
$$

For a clamped plate with initial displacement $w_{0}(r, \theta)$ and velocity $w_{1}(r, \theta)$, force and moment balancing yield the equations

$$
\begin{aligned}
& \left\{\begin{array}{cc}
\rho h \frac{\partial^{2} w}{\partial t^{2}}+\gamma \frac{\partial w}{\partial t}-\frac{\partial^{2} \mathcal{M}_{r}}{\partial r^{2}}-\frac{2}{r} \frac{\partial \mathcal{M}_{r}}{\partial r}+\frac{1}{r} \frac{\partial \mathcal{M}_{\theta}}{\partial r} & 0<\theta \leq 2 \pi \\
-\frac{2}{r} \frac{\partial^{2} \mathcal{M}_{r \theta}}{\partial r \partial \theta}-\frac{2}{r^{2}} \frac{\partial \mathcal{M}_{r \theta}}{\partial \theta}-\frac{1}{r^{2}} \frac{\partial^{2} \mathcal{M}_{\theta}}{\partial \theta^{2}}=\tilde{g}(t, r, \theta) & 0 \leq r<a
\end{array}\right. \\
& \begin{cases}w(t, a, \theta)=\frac{\partial w}{\partial r}(t, a, \theta)=0 & \\
\left\{w(0, r, \theta)=w_{0}(r, \theta) \quad, \quad \frac{\partial w}{\partial t}(0, r, \theta)=w_{1}(r, \theta) .\right.\end{cases}
\end{aligned}
$$

as the strong form of the plate model (see [9, 27] for details). From an applications perspective, the following observations can be made regarding this model.

1. It is first noted that in many applications, it is nearly impossible to maintain the truly fixed (zero displacement and slope) boundary conditions specified in the model (2.1). For the experimental plate which we used, parameter estimation results indicated minimal energy loss through the boundary conditions and an adequate fit of the model (2.1) was obtained (see the identification results in [2,3]). For plates in which the boundary clamping is less secure, a model for imperfectly clamped boundaries such as that presented in [13] should be used in order to obtain a model fit which is adequate for control applications. 
2. As discussed in [11], the plate parameters $\rho, D, \nu$ and $c_{D}$ are discontinuous due to the presence and differing material properties of the patches. Moreover, while 'handbook' values can be determined for $\rho, D$ and $\nu$ for a plate which is devoid of patches, those values usually cannot be used in the final system model with any accuracy due to nonuniformities in the plate and boundary conditions, variations in materials, and contributions due to the presence of the patches. In applications, such as that in this paper, these parameters are estimated using fit-to-data techniques (see $[2,3]$ for the parameter estimation results pertaining to the plate used here).

3. The input parameters $\mathcal{K}_{i}^{B}$ are discontinuous since they are nonzero only over the regions of the patches. While expressions for these constants are derived in [11], they too must be estimated in applications due to manufacturing variations in the patches.

It is readily noted in the strong form of the modeling equations that the discontinuous plate parameters and patch input terms are differentiated, thus leading to derivatives of the Dirac $\delta$ 'function'. The difficulties associated with this formulation are avoided in the weak form of the modeling equations which is presented in the next section.

\subsection{Weak Form of Plate Model}

To provide a framework which facilitates analysis, approximation and implementation, it is advantageous to consider a weak form of the modeling equations. Such a formulation can be determined directly from Hamiltonian (energy) principles and is equivalent to that obtained by integration by parts after multiplication of the strong form by suitably smooth test functions.

For the plate problem under consideration, a suitable space $V$ of test functions is the subset of the Sobolev space $H^{2}\left(\Gamma_{0}\right)$ which satisfies the essential boundary conditions $w=\frac{\partial w}{\partial r}=0$ at $r=a$. As detailed in $[9,29]$, a weak or variational form of the equations of motion for the plate is

$$
\begin{aligned}
\int_{\Gamma_{0}} \rho h \frac{\partial^{2} w}{\partial t^{2}} \bar{\eta} d \gamma+\int_{\Gamma_{0}} \gamma \frac{\partial w}{\partial t} \bar{\eta} d \gamma-\int_{\Gamma_{0}} M_{r} \frac{\overline{\partial^{2} \eta}}{\partial r^{2}} d \gamma-\int_{\Gamma_{0}} \frac{1}{r^{2}} M_{\theta}\left[r \overline{\frac{\partial \eta}{\partial r}}+\overline{\frac{\partial^{2} \eta}{\partial \theta^{2}}}\right] d \gamma \\
-2 \int_{\Gamma_{0}} \frac{1}{r^{2}} M_{r \theta}\left[r \overline{\frac{\partial^{2} \eta}{\partial r \partial \theta}}-\frac{\overline{\partial \eta}}{\partial \theta}\right] d \gamma=\int_{\Gamma_{0}} \sum_{i=1}^{s} \mathcal{K}_{i}^{B} u_{i}(t)_{\lambda_{i}}(r, \theta) \overline{\nabla^{2} \eta} d \gamma+\int_{\Gamma_{0}} \hat{g} \bar{\eta} d \gamma
\end{aligned}
$$

for all $\eta \in V$. The overbar here denotes complex conjugation and the differential is $d \gamma=r d \theta d r$. It is easily noted that in this form, derivatives that were originally applied to moments have been transferred onto test functions. This eliminates the difficulties associated with differentiating the piecewise constant parameters $\rho, D, \nu$ and $c_{D}$ found in the internal moments as well as the discontinuous input parameters $\mathcal{K}_{1}^{B}, \cdots, \mathcal{K}_{s}^{B}$.

\subsection{State Approximation}

As discussed in $[9,29]$, an appropriate choice for the basis and Fourier-Galerkin expansion of the plate displacement, when considering clamped boundary conditions, is $B_{k}^{\mathcal{N}}(r, \theta)=$ 
${ }^{|\hat{m}|} B_{n}^{m}(r) \epsilon^{i m \theta}$ and

$$
w^{\mathcal{N}}(t, r, \theta)=\sum_{m=-M}^{M} \sum_{n=1}^{N^{m}} w_{m n}^{\mathcal{N}}(t) r^{|\dot{m}|} B_{n}^{m}(r) \epsilon^{i m \theta}=\sum_{k=1}^{\mathcal{N}} w_{k}^{\mathcal{N}}(t) B_{k}^{\mathcal{N}}(r, \theta) .
$$

Here $B_{n}^{m}(r)$ is the $n^{\text {th }}$ modified cubic spline satisfying $B_{n}^{m}(a)=\frac{d B_{n}^{m}(a)}{d r}=0$ with the condition $\frac{d B_{n}^{m}(0)}{d r}=0$ being enforced when $m=0$ (this latter condition guarantees differentiability at the origin and implies that

$$
N^{m}= \begin{cases}N & , m=0 \\ N+1 & , m \neq 0\end{cases}
$$

where $N$ denotes the number of modified cubic splines). The total number of plate basis functions is $\mathcal{N}=(2 M+1)(N+1)-1$. As discussed in the [9, 29], the inclusion of the weighting term $r^{|\hat{m}|}$ with

$$
\hat{m}= \begin{cases}0 & , m=0 \\ 1 & , m \neq 0\end{cases}
$$

is motivated by the asymptotic behavior of the Bessel functions, which make up the analytic plate solution, as $r \rightarrow 0$. It also serves to ensure the uniqueness of the solution at the origin. The Fourier coefficient in the weight is truncated to control the conditioning of the mass and stiffness matrices (see the examples in [9]).

To obtain a matrix system (again, see $[9,29]$ for a careful derivation with complete details), the $\mathcal{N}$ dimensional approximating subspace is taken to be $H^{\mathcal{N}}=\operatorname{span}\left\{B_{k}^{\mathcal{N}}\right\}$ and the product space for the usual corresponding first-order vector system is $\mathcal{H}^{\mathcal{N}} \times \mathcal{H}^{\mathcal{N}}$. The restriction of the first-order form for the infinite-dimensional system (2.2) to the space $\mathcal{H}^{\mathcal{N}} \times \mathcal{H}^{\mathcal{N}}$ then yields the matrix equation

$$
\begin{aligned}
& {\left[\begin{array}{cc}
K_{D}^{\mathcal{N}} & 0 \\
0 & M^{\mathcal{N}}
\end{array}\right]\left[\begin{array}{l}
i^{\mathcal{N}}(t) \\
\ddot{\vartheta}^{\mathcal{N}}(t)
\end{array}\right]=\left[\begin{array}{cc}
0 & K_{D}^{\mathcal{N}} \\
-K_{D}^{\mathcal{N}} & -K_{c_{D}}^{\mathcal{N}}
\end{array}\right]\left[\begin{array}{c}
\vartheta^{\mathcal{N}}(t) \\
\dot{\vartheta}^{\mathcal{N}}(t)
\end{array}\right]+\left[\begin{array}{c}
0 \\
\tilde{B}^{\mathcal{N}}
\end{array}\right] u(t)+\left[\begin{array}{c}
0 \\
\hat{g}^{\mathcal{N}}(t)
\end{array}\right]} \\
& {\left[\begin{array}{cc}
K_{D}^{\mathcal{N}} & 0 \\
0 & M^{\mathcal{N}}
\end{array}\right]\left[\begin{array}{l}
\vartheta^{\mathcal{N}}(0) \\
\dot{\vartheta}^{\mathcal{N}}(0)
\end{array}\right]=\left[\begin{array}{c}
g_{1}^{\mathcal{N}} \\
g_{2}^{\mathcal{N}}
\end{array}\right]}
\end{aligned}
$$

where $\vartheta^{\mathcal{N}}(t)=\left[w_{1}^{\mathcal{N}}(t), w_{2}^{\mathcal{N}}(t), \cdots, w_{\mathcal{N}}^{\mathcal{N}}(t)\right]^{T}$ denotes the column $\mathcal{N}$ vector containing the approximate state coefficients (see (2.3)). The component matrices and vectors are given by

$$
\begin{aligned}
& \Lambda_{D}^{\mathcal{N}}=K_{D 1}+K_{D 2}+K_{D 3}+K_{D 4}+K_{D 5} \\
& \Lambda_{c_{D}}^{\mathcal{N}}=K_{c_{D} 1}+K_{C_{D} 2}+K_{c_{D} 3}+K_{c_{D} 4}+K_{c_{D} 5}+\int_{\Gamma_{0}} \gamma B_{k}^{\mathcal{N}} \overline{B_{\ell}^{\mathcal{N}}} d \gamma \\
& {\left[M^{\mathcal{N}}\right]_{\ell, k}=\int_{\Gamma_{0}} \rho h B_{k}^{\mathcal{N}} \overline{B_{\ell}^{\mathcal{N}}} d \gamma} \\
& {\left[\hat{g}^{\mathcal{N}}(t)\right]_{\ell}=\int_{\Gamma_{0}} \tilde{g} \overline{B_{\ell}^{\mathcal{N}}} d \gamma \quad, \quad\left[\tilde{B}^{\mathcal{N}}\right]_{\ell, j}=\int_{j^{t h p a t c h}} \mathcal{K}_{j} \overline{\nabla^{2} B_{\ell}^{\mathcal{N}}} d \gamma} \\
& {\left[g_{1}^{\mathcal{N}}\right]_{\ell}=\left\langle w_{0}, B_{\ell}^{\mathcal{N}}\right\rangle_{V}, \quad\left[g_{2}^{\mathcal{N}}\right]_{\ell}=\left\langle w_{0}, B_{\ell}^{\mathcal{N}}\right\rangle_{H}}
\end{aligned}
$$


where

$$
\begin{aligned}
& {\left[\kappa_{D 1}\right]_{\ell, k}=\int_{\Gamma_{0}} D\left[\frac{\partial^{2} B_{k}^{\mathcal{N}}}{\partial r^{2}}+\frac{\nu}{r} \frac{\partial B_{k}^{\mathcal{N}}}{\partial r}+\frac{\nu}{r^{2}} \frac{\partial^{2} B_{k}^{\mathcal{N}}}{\partial \theta^{2}}\right] \frac{\partial^{2} \overline{B_{r}^{\mathcal{N}}}}{\partial r^{2}} l_{\gamma},} \\
& {\left[\kappa_{D 2}\right]_{\ell, k}=\int_{\Gamma_{0}} D\left[\frac{1}{r^{2}} \frac{\partial B_{k}^{\mathcal{N}}}{\partial r}+\frac{1}{r^{3}} \frac{\partial^{2} B_{k}^{\mathcal{N}}}{\partial \theta^{2}}+\frac{\nu}{r} \frac{\partial^{2} B_{k}^{\mathcal{N}}}{\partial r^{2}}\right] \frac{\partial \overline{B_{\ell}^{\mathcal{N}}}}{\partial r} d \gamma,} \\
& {\left[K_{D 3}\right]_{\ell, k}=\int_{\Gamma_{0}} D\left[\frac{1}{r^{3}} \frac{\partial B_{k}^{\mathcal{N}}}{\partial r}+\frac{1}{r^{4}} \frac{\partial^{2} B_{k}^{\mathcal{N}}}{\partial \theta^{2}}+\frac{\nu}{r^{2}} \frac{\partial^{2} B_{k}^{\mathcal{N}}}{\partial r^{2}}\right] \frac{\partial^{2} \overline{B_{\ell}^{\mathcal{N}}}}{\partial \theta^{2}} d \gamma,} \\
& {\left[\mathrm{H}_{D 4}\right]_{\ell, k}=2 \int_{\Gamma_{0}} D(1-\nu)\left[\frac{1}{r^{2}} \frac{\partial^{2} B_{k}^{\mathcal{N}}}{\partial r \partial \theta}-\frac{1}{r^{3}} \frac{\partial B_{k}^{\mathcal{N}}}{\partial \theta}\right] \frac{\partial^{2} \overline{B_{\ell}^{\mathcal{N}}}}{\partial r \partial \theta} d \gamma,} \\
& {\left[K_{D 5}\right]_{\ell, k}=2 \int_{\Gamma_{0}} D(1-\nu)\left[-\frac{1}{r^{3}} \frac{\partial^{2} B_{k}^{\mathcal{N}}}{\partial r \partial \theta}+\frac{1}{r^{4}} \frac{\partial B_{k}^{\mathcal{N}}}{\partial \theta}\right] \frac{\partial \overline{B_{r}^{\mathcal{N}}}}{\partial \theta} d \gamma .}
\end{aligned}
$$

The index ranges here are $k, \ell=1, \cdots, \mathcal{N}$. The matrices $K_{C_{D} 1}-K_{C_{D} 5}$ are defined similarly with the inclusion of the parameter $c_{D}$ in the various integrals. Finally, we remind the reader that $\rho, D, \nu$ and $c_{D}$ are piecewise constant in these definitions due to the presence of the patches.

For application purpose, it useful to note that the matrix system for the plate can thus be written as the Cauchy system

$$
\begin{aligned}
& \dot{y}^{\mathcal{N}}(t)=A^{\mathcal{N}} y^{\mathcal{N}}(t)+B^{\mathcal{N}} u(t)+g^{\mathcal{N}}(t) \\
& y^{\mathcal{N}}(0)=y_{0}^{\mathcal{N}}
\end{aligned}
$$

where $y^{\mathcal{N}}(t)=\left[\vartheta^{\mathcal{N}}(t), i^{\mathcal{N}}(t)\right]^{T}=\left[w_{1}^{\mathcal{N}}(t), \cdots, w_{\mathcal{N}}^{\mathcal{N}}(t), \dot{w}_{1}^{\mathcal{N}}(t), \cdots, \dot{w}_{\mathcal{N}}^{\mathcal{N}}(t)\right]^{T}$ denotes the column $2 \mathcal{N}$ vector containing the generalized Fourier coefficients for the approximate displacement and velocity. In this form, the control problem can be readily discussed.

\section{Continuous Time Control Problem}

In the preceding discussion leading from the infinite dimensional PDE model to the finite dimensional matrix approximation, the superscript $\mathcal{N}$ was used to denote the level of discretization; i.e., the number of Fourier/spline basis elements used to approximate the state. This notation is standard in the theory of finite element and spline approximations of infinite dimensional systems. In finite dimensional control theory, however, the level of discretization is typically fixed and these subscript $\mathcal{N}$ 's are usually omitted to simplify notation. We will do the same in this and subsequent sections so as to remain consistent with standard control notation.

\subsection{Initial Displacement and Velocity - LQG Control Law}

We consider first the $\mathcal{N}$-dimensional systems

$$
\begin{aligned}
& \dot{y}(t)=A y(t)+B u(t) \quad, \quad y(0)=y_{0} \\
& y_{o b}(t)=C y(t) \\
& z(t)=H y(t)+C u(t)
\end{aligned}
$$


where $y_{o b}$ denotes observations in $\mathbb{R}^{P}$ and $C$ is a $P \times \mathcal{N}$ observation matrix whose structure is determined by the manner and number of observations being used (the specific $C$ matrix used in the plate experiments is described in Section 4.2). Moreover, $\approx \in \mathbb{R}^{r}$ denotes the performance output obtained under the assumption that $G$ and $H$ are time invariant matrices satisfying $H^{T} G=0$. In the event that $P=\mathcal{N}$ and $C$ is an identity, the optimal control $u$ can be obtained from standard linear quadratic regulator (LQR) optimal control theory. The number of observations $P$ is usually limited, however, and we concentrate instead on the case $P<\mathcal{N}$ which occurs when the full state is unavailable and must be reconstructed using a compensator (e.g., see $[25])$.

The general control problem for this case consists of determining the voltage $u$ which minimizes the performance index (or cost functional)

$$
\begin{aligned}
J(u) & =\int_{0}^{\infty}|z(t)|^{2} d t \\
& =\int_{0}^{\infty}\{\langle Q y(t), y(t)\rangle+\langle R u(t), u(t)\rangle\} d t
\end{aligned}
$$

subject to (3.1). The $\mathcal{N} \times \mathcal{N}$ matrix $Q$ can be chosen to satisfy various design criteria including frequency windowing, the weighting of various state components, or minimization of certain energy measures. The $Q$ matrix used here was chosen using energy considerations and construction details can be found in Section 4.2. The $s \times s$ matrix $R$ weights the voltages to the various patches or patch pairs.

Because full state information is not available in most applications, the state must be estimated or reconstructed from observations before a controlling voltage can be determined. We consider here a full order compensator or observer of Luenberger type [25] and refer the reader to $[16,22]$ for details on reduced order observers.

The compensator or reconstructed state satisfies the matrix system

$$
\begin{aligned}
& \dot{y}_{c}(t)=A y_{c}(t)+B u(t)+F\left[y_{o b}(t)-C y_{c}(t)\right] \\
& y_{c}(0)=y_{c_{0}}
\end{aligned}
$$

with the optimal voltage

$$
u(t)=-K y_{c}(t)
$$

where $F$ and $K$ denote the compensator and feedback gains, respectively. We note that $K^{r}$ and $F$ are chosen so that the reconstruction error $\left|y(t)-y_{c}(t)\right| \rightarrow 0$ as $t \rightarrow \infty$. Under usual observability and controllability hypotheses (see [25]), the optimal feedback and compensator gains are given by

$$
\begin{aligned}
& I=R^{-1} B^{T} \Pi \\
& F=P C^{T} \dot{R}^{-1}
\end{aligned}
$$

where $\Pi$ and $P$ are unique nonnegative-definite solutions to the following feedback (regulator) and compensator (observer) algebraic Riccati equations

$$
\begin{aligned}
& \Pi A+A^{T} \Pi-\Pi B R^{-1} B^{T} \Pi+Q=0 \\
& P A^{T}+A P-P C^{T} \tilde{R}^{-1} C P+\tilde{Q}=0,
\end{aligned}
$$


respectively. As was the case with the matrices $Q$ and $R$, the matrices $\dot{Q}$ and $\tilde{R}$ are design criteria for the specific control application under consideration (specific choices used in the plate experiments are summarized in Section 4.2). We point out that in terms of the component matrices and control voltage, the compensator can be expressed as

$$
\dot{y}_{c}(t)=\left[A-B R^{-1} B^{T} \Pi\right] y_{c}(t)+P C^{T} \dot{R}^{-1} C\left[y(t)-y_{c}(t)\right]
$$

The control law just described must be implemented in real time in order to be a viable method for reducing vibrations in physical structures. To facilitate implementation, it is prudent to calculate offline as many components as possible and then treat those pre-calculated components as filters when performing online computations. The method for continuous time implementation is summarized and offline and online components are categorized in the following algorithm.

\section{Algorithm 3.1.1: Continuous Time Control of Initial Displacement and Velocity}

\begin{tabular}{|c|c|c|}
\hline Offline & $\begin{array}{l}\text { (i) } \\
\text { (ii) } \\
\text { (iii) }\end{array}$ & $\begin{array}{l}\text { Construct matrices } A, B, C, Q, R, \dot{Q}, \tilde{R} \\
\text { Solve Riccati equations }(\mathbf{3 . 3}) \text { for } \Pi \text { and } P \\
\text { Construct } K=R^{-1} B^{T} \Pi \text { and } F=P C^{T} \dot{R}^{-1}\end{array}$ \\
\hline Online & $\begin{array}{l}(i) \\
(i i)\end{array}$ & $\begin{array}{l}\text { Collect data } y_{o b}(t)=C y(t) \\
\text { Solve the ODE system } \\
\begin{aligned} \dot{y}_{c}(t)=[A-B I-F C] y_{c}(t)+F y_{o b}(t) \\
\quad=A_{c} y_{c}(t)+F y_{o b}(t)\end{aligned} \\
\text { Calculate the voltage } u(t)=-K y_{c}(t)\end{array}$ \\
\hline
\end{tabular}

We note that the expensive (time consuming) calculation of the component matrices $A, B, Q, R$, $C, \hat{Q}, \hat{R}$ and Riccati solutions $\Pi, P$ is performed offline with the results loaded into the control code as datafiles. This leaves the integration of the system $\dot{y}_{c}(t)=A_{c} y_{c}(t)+F y_{o b}(t)$ as the primary computation to be performed during implementation. Issues regarding the numerical integration of the system as well as the effects of discrete time calculations will be discussed in Section 4, and a discrete version of Algorithm 3.1.1 is summarized in Algorithm 4.1.1. Finally, the observed system, performance index and state estimator and LQG control law for the system are summarized in Algorithm 3.4.1 in Section 3.4.

While this compensator does provide the desired performance, it may lack robustness in some applications. In cases where added robustness with regard to certain types of system or observation noise and modeling errors is required, an $H^{\infty} /$ MinMax compensator of the type described in the next section can be used. 


\subsection{Initial Displacement and Velocity $-H^{\infty} /$ MinMax Control Law}

We consider now the design of a dynamic compensator which is robust with respect to certain types of state and measurement uncertainties or disturbances (see [15] for details). To incorporate such uncertainties, we let $w(t) \in \mathbb{R}^{q}$ denote input and output disturbances. The system, with no exogenous force, is then given by

$$
\begin{aligned}
& \dot{y}(t)=A y(t)+B u(t)+D w(t) \\
& y_{o b}(t)=C y(t)+E w(t) \\
& z(t)=H y(t)+G u(t) .
\end{aligned}
$$

For this discussion, we assume that the input and output disturbance are independent and hence $D E^{T}=0$. This is a matter of convenience and the dependent case can be handled similarly after slight modifications are made (see [15]).

In this case, the min/max optimization problem leading to the controller consists of finding a controller $u^{*} \in U \equiv L^{2}\left(0, \infty ; \mathbb{R}^{s}\right)$ and disturbance $w^{*} \in W \equiv L^{2}\left(0, \infty ; \mathbb{R}^{q}\right)$ such that

$$
J_{\gamma}^{*}=\inf _{u \in U^{I}} \sup _{w \in W} J_{\gamma}(u, w)=J_{\gamma}\left(u^{*}, w^{*}\right)
$$

for the disturbance augmented functional

$$
\begin{aligned}
J_{\gamma}(u, w) & =\int_{0}^{\infty}\left\{|\tilde{\sim}(t)|^{2}-\gamma^{2}|w(t)|^{2}\right\} d t \\
& =\int_{0}^{\infty}\left\{\langle Q y(t), y(t)\rangle+\langle R u(t), u(t)\rangle-\gamma^{2}\langle w(t), w(t)\rangle\right\} d t .
\end{aligned}
$$

As noted in $[12,15]$, the results from this optimization problem yield a bound $\gamma$ for the $H^{\infty}$ norm of the transfer function from disturbance $\mathcal{L}(w)$ to the performance output $\mathcal{L}(z)$ where $\mathcal{L}$ denotes the Laplace transform.

Under the assumption that the pair $(A, B)$ is stabilizable, $(A, C)$ is detectable, $(A, G)$ is controllable, and $(A, H)$ is observable, one can prove the existence of (minimal) positive definite solutions $\Pi$ and $P$ to the algebraic Riccati equations

$$
\begin{aligned}
& \Pi A+A^{T} \Pi-\Pi\left[B R^{-1} B^{T}-\gamma^{-2} \hat{Q}\right] \Pi+Q=0 \\
& P A^{T}+A P-P\left[C^{T} \tilde{R}^{-1} C-\gamma^{-2} Q\right] P+\dot{Q}=0
\end{aligned}
$$

for a given attenuation $\gamma>0$. Moreover, if the spectral radius $\rho$ of $P \Pi$ satisfies the condition

$$
\rho(P \Pi)<\gamma^{2}, \quad \text { or } \quad \Pi-\gamma^{2} P^{-1}<0,
$$

then there exists a unique optimal controller

$$
u^{*}(t)=-R^{-1} B^{T} \Pi y_{c}(t) .
$$

The state estimator $y_{c}(t) \in \mathbb{R}^{\mathcal{N}}$ satisfies

$$
\begin{aligned}
& \dot{y}_{c}(t)=A_{c} y_{c}(t)+F y_{o b}(t) \\
& y_{c}(0)=y_{c_{0}}
\end{aligned}
$$


where

$$
\begin{aligned}
& A_{c}=A-B \Lambda-F C+\gamma^{-2} \check{Q} \Pi \\
& F=\left[I-\gamma^{-2} P \Pi\right]^{-1} P C^{T} \tilde{R}^{-1} .
\end{aligned}
$$

The implementation issues concerning the method are similar to those discussed in the algorithm for the LQG controller but determination of the Riccati solutions $\Pi, P$ and filter $F$ are complicated by the fact that a suitable design value of $\gamma$ must be determined before matrix calculations can proceed. Fortunately, these calculations can be performed offline and resulting matrices input as data files for the online computations. Hence the actual online controller can run at the same rate as that obtained using the LQG methodology.

The form of the observed system, the extended performance index and the $H^{\infty} / \mathrm{MinMax}$ control law are summarized in Algorithm 3.4.2 in Section 3.4. The various components can be compared with the LQG components summarized previously as well as those which arise when a periodic exogenous force is considered.

\subsection{Periodic Primary Excitation}

For the case in which a periodic exogenous force drives the system, knowledge of that force can be used extend previously discussed results to include the effects of periodicity. The $\mathcal{N}$ dimensional system in this case is

$$
\begin{aligned}
& \dot{y}(t)=A y(t)+B u(t)+g(t) \quad, \quad y(0)=y(\tau) \\
& y_{o b}(t)=C y(t)
\end{aligned}
$$

where $g(t) \in \mathbb{R}^{\mathcal{N}}$ is periodic with period $\tau$. That periodicity is then reflected in the performance index

$$
J(u)=\int_{0}^{\tau}\{\langle Q y(t), y(t)\rangle+\langle R u(t), u(t)\rangle\} d t
$$

which is minimized subject to (3.4).

With $K$ and $F$ defined in (3.2), the reconstructed state in this case satisfies the system

$$
\begin{aligned}
& \dot{y}_{c}(t)=A y_{c}(t)+B u(t)+F\left[y_{o b}(t)-C y_{c}(t)\right]+g(t) \\
& y_{c}(0)=y_{c}(\tau)
\end{aligned}
$$

with the optimal voltage given by

$$
u(t)=-K_{c}(t)+R^{-1} B^{T} r(t)
$$

Here $r$ is a tracking variable defined by the system

$$
\begin{aligned}
& \dot{r}(t)=-\left[A-B K^{r}\right]^{T} r(t)+\Pi g(t) \\
& r(0)=r(\tau)
\end{aligned}
$$

where $\Pi$ solves the first of the algebraic Riccati equations (3.3). We point out that in this case, the voltage contains two contributions. The first incorporates transient information by 
feeding back state estimates while the tracking component incorporates information regarding the periodic force.

Combining (3.5) and (3.6) yields the single expression

$$
\begin{aligned}
& \dot{y}_{c}(t)=\left[A-B K^{\prime}\right] y_{c}(t)+F C^{\prime}\left[y(t)-y_{c}(t)\right]+B R^{-1} B^{T} r(t)+g(t) \\
& y_{c}(0)=y_{c}(\tau) .
\end{aligned}
$$

for the state estimator in terms of the tracking variable. The state estimate at time $t$ is then obtained by integrating (3.7) and (3.8) after solving the necessary Riccati equations.

As was the case when considering control of the unforced system with initial displacement and velocity, the computations can be categorized with respect to those which can be performed offline and those which must be done online. The following algorithm summarizes the continuous time control method for periodic excitation and categorizes the offline and online components.

\section{Algorithm 3.3.1: Continuous Time Control with Periodic Exogenous Force}

\begin{tabular}{|l} 
Offline \\
(i)
\end{tabular}

In this case, the tracking equation $\dot{r}(t)=A_{t r} r(t)+\hat{g}(t)$ must be solved before the state can be estimated by integrating the system $\dot{y}_{c}(t)=A_{c} y_{c}(t)+F y_{o b}(t)+A_{r} r(t)+g(t)$. Details regarding the solution of these two systems with discrete data and force measurements are given in Algorithm 4.1.2 in the next section.

Finally, the governing equations and component matrices for the LQG controller are summarized in Algorithm 3.4.3 of Section 3.4. Expressions similar to those in Section 3.2 arise when an $H^{\infty}$ /MinMax compensator is considered for the problem and the resulting control law is summarized in Algorithm 3.4.4. We also refer the reader to [4] where details concerning the design of an $H^{\infty} /$ MinMax compensator for a structural acoustic system that is subjected to a. periodic exogenous force is considered. 


\subsection{Summary of Systems, Performance Indices and Control Laws}

The modeling state equations, observed system, performance index and compensator design for the previously discussed cases are summarized in this section. Specifically, the LQG and $H^{\infty} /$ MinMax control laws are summarized for the system with no primary force as well as the system subjected to a periodic exogenous force. Details regarding the construction of the individual components can be found in the preceding section or in the implementation section which follows.

\section{Algorithm 3.4.1: LQG Control Law for Unforced System}

(1) Observed System

$$
\begin{aligned}
& \dot{y}(t)=A y(t)+B u(t) \\
& y_{o b}(t)=C y(t) \\
& \tilde{z}(t)=I I y(t)+G u(t)
\end{aligned}
$$

$y \in \mathbb{R}^{\mathcal{N}}, u \in \mathbb{R}^{s}$

$y_{o b} \in \mathbb{R}^{p}$

$\tilde{z} \in \mathbb{R}^{r}, H^{T} G=0$

(2) Performance Index

$$
\begin{aligned}
J(u) & =\int_{0}^{\infty}|\tilde{z}(t)|^{2} d t \\
& =\int_{0}^{\infty}\{\langle Q y(t), y(t)\rangle+\langle R u(t), u(t)\rangle\} d t \quad Q=H^{T} H, R=G^{T} G
\end{aligned}
$$

(3) State Estimator and Control Law

$$
\begin{aligned}
& \dot{y}_{c}(t)=A_{c} y_{c}(t)+F y_{o b}(t) \\
& A_{c}=A-F C-B K \\
& K=R^{-1} B^{T} \Pi \\
& F=P C^{T} \tilde{R}^{-1} \\
& \Pi A+A^{T} \Pi-\Pi B R^{-1} B^{T} \Pi+Q=0 \\
& P A^{T}+A P-P C^{T} \tilde{R}^{-1} C P+\tilde{Q}=0
\end{aligned}
$$

Controlling Voltage

$u(t)=-H_{c}(t)$ 


\section{Algorithm 3.4.2: $H^{\infty} /$ MinMax Control Law for Unforced System}

(1) Observed System

$$
\begin{aligned}
& \dot{y}(t)=A y(t)+B u(t)+D w(t) \\
& y_{o b}(t)=C y(t)+E w(t) \\
& z(t)=H y(t)+C u(t)
\end{aligned}
$$$$
y \in \mathbb{R}^{\mathcal{N}}, u \in \mathbb{R}^{s}, w \in \mathbb{R}^{q}
$$$$
y_{o b} \in \mathbb{R}^{p}, D E^{T}=0
$$$$
\approx \in \mathbb{R}^{r}, H^{T} G^{\prime}=0
$$$$
Q=H^{T} H \geq 0, R=G^{T} G>0
$$$$
\tilde{Q}=D D^{T} \geq 0, \hat{R}=E E^{T}>0
$$

(2) Performance Index

$$
\begin{aligned}
J_{\gamma}(u, w) & =\int_{0}^{\infty}\left\{|z(t)|^{2}-\gamma^{2}|w(t)|^{2}\right\} d t \\
& =\int_{0}^{\infty}\left\{\langle Q y(t), y(t)\rangle+\langle R u(t), u(t)\rangle-\gamma^{2}\langle w(t), w(t)\rangle\right\} d t
\end{aligned}
$$

(3) State Estimator and Control Law

$$
\begin{aligned}
& \dot{y}_{c}(t)=A_{c} y_{c}(t)+F y_{o b}(t) \\
& A_{c}=A-F C-B{ }^{\prime}+\gamma^{-2} \dot{Q} \Pi \\
& K=R^{-1} B^{T} \Pi \\
& F=\left[I-\gamma^{-2} P \Pi\right]^{-1} P C^{T} \tilde{R}^{-1} \\
& \Pi A+A^{T} \Pi-\Pi\left[B R^{-1} B^{T}-\gamma^{-2} \hat{Q}\right] \Pi+Q=0 \\
& P A^{T}+A P-P\left[C^{T} \tilde{R}^{-1} C-\gamma^{-2} Q\right] P+\hat{Q}=0
\end{aligned}
$$

\section{Controlling Voltage}

$$
u(t)=-\kappa y_{c}(t)
$$

Note: $\rho(P \Pi)<\gamma^{2}$ or $\Pi-\gamma^{2} P^{-1}<0$ 


\section{Algorithm 3.4.3: LQG Control Law for Periodic Exogenous Force}

(1) Observed System

$$
\begin{array}{ll}
\dot{y}(t)=A y(t)+B u(t)+g(t) & y \in \mathbb{R}^{\mathcal{N}}, u \in \mathbb{R}^{s} \\
y(0)=y(\tau) & \\
y_{o b}(t)=C y(t) & y_{o b} \in \mathbb{R}^{p} \\
z(t)=H y(t)+G u(t) & z \in \mathbb{R}^{r}, H^{T} G=0
\end{array}
$$

(2) Performance Index

$$
\begin{aligned}
J(u) & =\int_{0}^{T}|z(t)|^{2} d t \\
& =\int_{0}^{\tau}\{\langle Q y(t), y(t)\rangle+\langle R u(t), u(t)\rangle\} d t \quad Q=H^{T} H, R=G^{T} G^{t}
\end{aligned}
$$

(3) State Estimator and Control Law

$$
\begin{aligned}
& \dot{y}_{c}(t)=A_{c} y_{c}(t)+F y_{o b}(t)+A_{r} r(t)+g(t) \\
& A_{c}=A-F C-B K \\
& A_{r}=B R^{-1} B^{T} \\
& K=R^{-1} B^{T} \Pi \\
& F=P C^{T} \tilde{R}^{-1} \\
& \Pi A+A^{T} \Pi-\Pi B R^{-1} B^{T} \Pi+Q=0 \\
& P A^{T}+A P-P C^{T} \tilde{R}^{-1} C P+\dot{Q}=0
\end{aligned}
$$

Tracking Equation

$$
\begin{aligned}
& \dot{r}(t)=A_{t r} r(t)+\hat{g}(t) \\
& A_{t r}=-\left[A-B K^{\prime}\right]^{T} \\
& \hat{g}(t)=\Pi g(t)
\end{aligned}
$$

Control Voltage

$$
u(t)=-\Lambda y_{c}(t)+R^{-1} B^{T} r(t)
$$




\section{Algorithm 3.4.4: $H^{\infty} /$ MinMax Control Law for Periodic Exogenous Force}

(1) Observed System

$$
\begin{array}{ll}
\dot{y}(t)=A y(t)+B u(t)+D w(t)+g(t) & y \in \mathbb{R}^{\mathcal{N}}, u \in \mathbb{R}^{s}, w \in \mathbb{R}^{q} \\
y(0)=y(\tau) & \\
y_{o b}(t)=C y(t)+E w(t) & y_{o b} \in \mathbb{R}^{p}, D E^{T}=0 \\
z(t)=H y(t)+C u(t) & z \in \mathbb{R}^{r}, H^{T} G=0 \\
& Q=H^{T} H \geq 0, R=G G^{T} G>0 \\
& \dot{Q}=D D^{T} \geq 0, R=E E^{T}>0
\end{array}
$$

(2) Performance Index

$$
\begin{aligned}
J_{\gamma}(u, w) & =\int_{0}^{\tau}\left\{|z(t)|^{2}-\gamma^{2}|w(t)|^{2}\right\} d t \\
& =\int_{0}^{\tau}\left\{\langle Q y(t), y(t)\rangle+\langle R u(t), u(t)\rangle-\gamma^{2}\langle w(t), w(t)\rangle\right\} d t
\end{aligned}
$$

(3) State Estimator and Control Law

$\dot{y}_{c}(t)=A_{c} y_{c}(t)+F y_{o b}(t)+A_{r} r(t)+g(t)$

$A_{c}=A-F C-B K+\gamma^{-2} \check{Q} \Pi$

$A_{r}=B R^{-1} B^{T}-\gamma^{-2} \tilde{Q}$

$K=R^{-1} B^{T} \Pi$

$F=\left[I-\gamma^{-2} P \Pi\right]^{-1} P C^{T} \tilde{R}^{-1}$

$\Pi A+A^{T} \Pi-\Pi\left[B R^{-1} B^{T}-\gamma^{-2} \tilde{Q}\right] \Pi+Q=0$

$P A^{T}+A P-P\left[C^{T} \tilde{R}^{-1} C-\gamma^{-2} Q\right] P+\tilde{Q}=0$

Tracking Equation

$\dot{r}(t)=A_{t r} r(t)+\hat{g}(t)$

$A_{t r}=-\left[A-B K-\gamma^{-2} \grave{Q} \Pi\right]^{T}$

$\hat{g}(t)=\Pi g(t)$

\section{Control Voltage}

$$
u(t)=-K^{r} y_{c}(t)+R^{-1} B^{T} r(t)
$$

Note: $\rho(P \Pi)<\gamma^{2}$ or $\Pi-\gamma^{2} P^{-1}<0$ 


\section{Discrete Time Control Problem}

The control laws summarized in the last section were derived under the assumption of continuous time sampling of observed state and force data. Hence it was assumed that $y_{o b}(t)$ and $g(t)$ were available for all $t$ within the temporal interval of interest. Moreover, it was assumed that $y(t), y_{c}(t)$ and $r(t)$ could be obtained through exact integration of the state, state estimator and tracking equations. When implementing the method, however, one has available only discrete data values and the differential estimator and tracking equations must be numerically approximated at discrete time values. The manner in which the control laws are implemented in discrete time and the influence of this discretization on the overall performance are discussed in this section.

In the last section, it was demonstrated that in the case with no primary exogenous force, the state equation, state estimator and controlling voltage for the $\mathrm{LQG}$ and $H^{\infty} / \mathrm{MinMax}$ problems had the form summarized in column 1 of Table 4.1. The corresponding quantities for a system subjected to a periodic exogenous force are summarized in the second column. Details regarding the component matrices can be found in the previous section.

\begin{tabular}{l|l|l} 
& \multicolumn{1}{|c|}{ No Primary Input } & \multicolumn{1}{c}{ Periodic Exogenous Force } \\
\hline State & $\dot{y}(t)=A y(t)+B u(t), y(0)=y_{0}$ & $\dot{y}(t)=A y(t)+B u(t)+g(t), y(0)=y(\tau)$ \\
Equation & $y_{o b}(t)=C y(t)$ & $y_{o b}(t)=C y(t)$ \\
\hline State & $\dot{y}_{c}(t)=A_{c} y_{c}(t)+F y_{o b}(t)$ & $\dot{y}_{c}(t)=A_{c} y_{c}(t)+F y_{o b}(t)+A_{r} r(t)+g(t)$ \\
Estimator & $y_{c}(0)=y_{c_{0}}$ & $y_{c}(0)=y_{c}(\tau)$ \\
\hline Tracking & & $\dot{r}(t)=A_{t r} r(t)+g(t)$ \\
Equation & & $r(0)=r(\tau)$ \\
\hline Control & $u(t)=-R^{-1} B^{T} \Pi y_{c}(t)$ & $u(t)=-R^{-1} B^{T} \Pi y_{c}(t)+R^{-1} B^{T} r(t)$ \\
Voltage & &
\end{tabular}

Table 4.1. State, state estimator and controlling voltage for the systems with no primary input and a periodic exogenous force.

The relationship of these components to the experimental plate setup, driven by a periodic exogenous force, is illustrated in Figure 4.1. For that setup, the physical process consists of the clamped circular plate with attached piezoceramic patches. The state equations represent the spatial discretization of a PDE model of the process with physical parameters estimated using experimental data so that the model accurately captures the plate dynamics. 


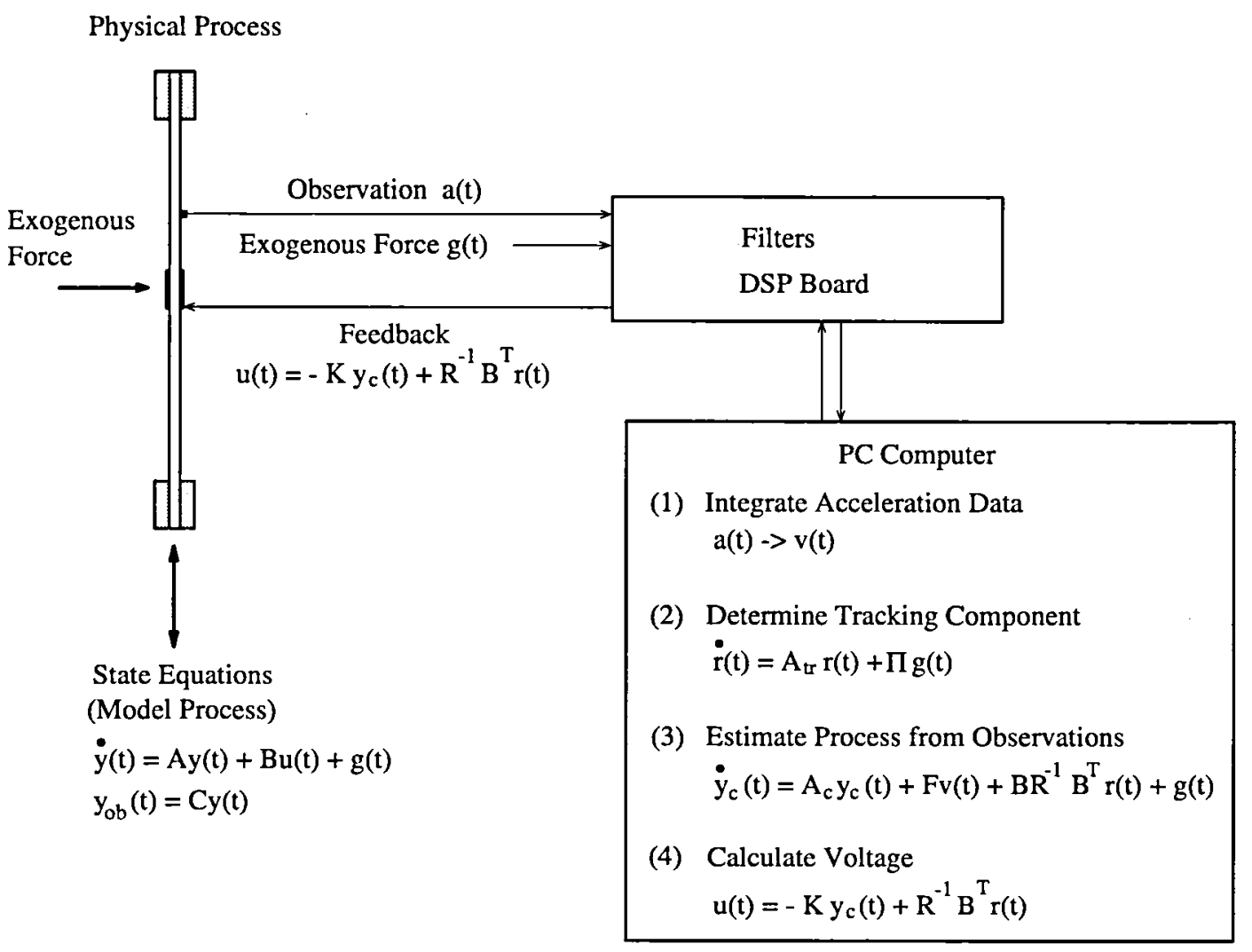

Figure 4.1. Experimental plate setup, modeling equations and computations necessary for determination of controlling voltage.

We point out that when modeling the process or performing simulations, the observations $y_{o b}(t)$ are obtained from the approximate state through the relation $y_{o b}(t)=C y(t)$ where $C$ is the matrix observation operator. When experimentally implementing the method, temporal data from the process is used to determine the observations $y_{o b}(t)$. This often requires processing either through digital or analog filters or in the software. In the circular plate experiments, accelerometer data $a(t)$ was integrated to yield velocity measurements $v(t)$ to be used for the observations $y_{o b}(t)$ (see the final subsection of this section for details). Once $y_{o b}(t)$ has been obtained, the state is estimated by numerically integrating the estimator equation and a controlling voltage is calculated. This voltage is then fed back to the process.

Because data is processed in a digital manner, the observations $y_{o b}$ and force measurements $g$ are obtained only at discrete times $t_{j}$. The rate at which this data can be sampled is governed by the data acquisition system, the software being used and the number of calculations required between samples. In particular, the tracking component $r\left(t_{j}\right)$, state estimate $y_{c}\left(t_{j}\right)$ and voltage $u\left(t_{j}\right)$ must be calculated before the arrival of data at time $t_{j+1}$. While details regarding these calculations are postponed until Section 4.1, it is noted here that the system sizes must be minimized in order to permit real-time approximation of the estimation equation. This is a major motivation for choosing appropriate, accurate approximation techniques to spatially discretize the modeling PDE's.

As indicated previously, the open and closed loop process dynamics can be simulated by using the state equations to model the plate. The state estimate and state are then approxi- 
mated by simultaneously integrating the corresponding equations. The integration of the state equation can be performed using any sufficiently accurate ODE routine which is efficient for the system under consideration; for example, a variable order, variable stepsize method was used to solve the stiff system which arose when simulating plate dynamics. If simulations demonstrating the levels of control that can be obtained under "optimal" conditions are desired, the estimator equation can be integrated using the same high-order routine. On the other hand, simulations representing the attenuation levels that can be expected under "implementation" conditions can be obtained by incorporating values of $y_{o b}$ and $g$ calculated at discrete times and approximating $y_{c}\left(t_{j}\right)$ using the techniques employed when implementing the method. Simulation results using both techniques can be found in [14].

\subsection{Approximation of the Estimator and Tracking Equations}

In order to obtain tracking values and state estimates to be used when calculating controlling voltages, the solution to the tracking and state estimator equations must be numerically approximated. If the goal is solely to perform simulations, this can be easily accomplished using the same ODE solver used to integrate the state equation (indeed, the state and estimator equations can be combined into a single system and integrated simultaneously). This is not practical when experimentally implementing the method, however, and one must typically perform the work subject to the following criteria. The method must be sufficiently efficient so as to facilitate real-time implementation and sufficiently accurate so as to resolve system dynamics. The systems are quite often stiff which implies that either $a$-stability or $a$-stability is important. Finally, the difficulties in storing past data make it prohibitive to use many popular multistep methods.

For the experiments performed with the circular plate, the sample rate was sufficiently fast (and hence $\Delta t$ was sufficiently small) that a modified backward Euler or trapezoidal method produced adequate results. We illustrate here such a modified backward Euler method.

\subsubsection{Initial Displacement and Velocity}

Considering first the compensator/estimator system with no primary exogenous force, we find that the modified Euler approximate to the solution at time $t_{j+1}$ is given by

$$
\begin{aligned}
y_{c_{j+1}} & =\left(I-\Delta t A_{c}\right)^{-1} y_{c_{j}}+\left(I-\Delta t A_{c}\right)^{-1} F y_{o b}\left(t_{j}\right) \\
& =\mathcal{A}_{c} y_{c_{j}}+\mathcal{F}_{c} y_{o b}\left(t_{j}\right) .
\end{aligned}
$$

The method is modified in the sense that current observation values $y_{o b}\left(t_{j}\right)$ are used as input since futures values at $t_{j+1}$ are unknown. The time step $\Delta t$ is dictated by the sample rate. We point out that the matrix $\mathcal{A}_{c}=\left(I-\Delta t A_{c}\right)^{-1}$ and vector $\mathcal{F}_{c}=\left(I-\Delta t A_{c}\right)^{-1} F$ can be computed offline and then loaded as datafiles for the online computations. Hence the implicit nature of the method, which is necessary to ensure stability, does not slow the implementation. The discrete time implementation of the method is summarized in Algorithm 4.1.1 which follows. The definitions of the component matrices can be found in Section 3, and Algorithm 4.1.1 can be compared with the corresponding continuous-time Algorithm 3.1.1 given in that section. 


\section{Algorithm 4.1.1: Discrete Time Control of Initial Displacement and Velocity}

\begin{tabular}{|c|c|c|}
\hline Offline & $\begin{array}{l}\text { (i) } \\
(i i) \\
(i i i) \\
(i v) \\
(v)\end{array}$ & 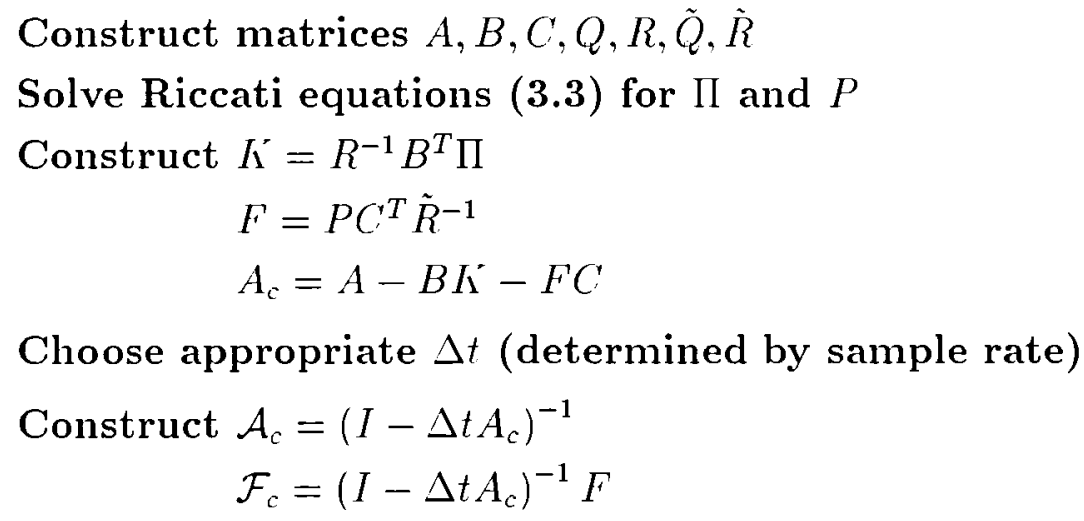 \\
\hline Online & $\begin{array}{l}\text { (i) } \\
\text { (ii) } \\
\text { (iii) } \\
\text { (iv) }\end{array}$ & $\begin{array}{l}\text { Collect acceleration data } a\left(t_{j}\right) \\
\text { Integrate to obtain } y_{o b}\left(t_{j}\right)=v\left(t_{j}\right) \\
\text { Time step the discrete estimator system } \\
\qquad y_{c_{j+1}}=\mathcal{A}_{c} y_{c_{j}}+\mathcal{F}_{c} y_{o b}\left(t_{j}\right) \\
\text { Calculate the voltage } u\left(t_{j}\right)=-K y_{c_{j}}\end{array}$ \\
\hline
\end{tabular}

\subsubsection{Periodic Exogenous Force}

The application of these control techniques to systems with both transient and steady state beliavior involves the approximation of both the tracking and state estimator equations before a control input is calculated. While a variety of techniques and strategies can be used to obtain approximate values of $r\left(t_{j}\right)$, which are then used when computing $y\left(t_{j}\right)$, these calculations must ultimately be performed in real time when implementing the method. In the experiments involving the circular plate, the exogenous force was measured for several periods and the solutions to the tracking equation were approximated and stored over a time period commensurate with the driving frequency. These stored tracking values were then used as a filter when approximating the estimated state during the remainder of the experiment.

Illustrating with the backward Euler discretization, the approximate to the tracking solution was determined from the difference equation

$$
\begin{aligned}
r_{j+1} & =\left(I-\Delta t A_{t r}\right)^{-1} r_{j}+\left(I-\Delta t A_{t r}\right)^{-1} \hat{g}\left(t_{j}\right) \\
& =\mathcal{A}_{t r} r_{j}+\mathcal{A}_{t r} \hat{g}\left(t_{j}\right)
\end{aligned}
$$

subject to the final condition $r(\tau)=0$ (when implementing the method, one can simply search for a "suitable" zero crossing to start the approximation).

This approximation was continued throughout several periods of the driving force with $r_{j}$ being stored in a circular buffer. This buffer was then treated as a filter when estimating the state using the difference equations

$$
\begin{aligned}
y_{c_{j}+1} & =\left(I-\Delta t A_{c}\right)^{-1} y_{c_{j}}+\left(I-\Delta t A_{c}\right)^{-1} F y_{o b}\left(t_{j}\right)+\left(I-\Delta t A_{c}\right)^{-1} A_{r} r_{j}+\left(I-\Delta t A_{c}\right)^{-1} g\left(t_{j}\right) \\
& =\mathcal{A}_{c} y_{c_{j}}+\mathcal{F}_{c} y_{o b}\left(t_{j}\right)+\mathcal{A}_{r} r_{j}+\mathcal{A}_{c} g\left(t_{j}\right) .
\end{aligned}
$$


As indicated in the following algorithm, the time intensive calculations involving matrix construction, solution of the Riccati equations and matrix inversion were performed offline prior to the experiments, and the matrices $\mathcal{A}_{t r}, \mathcal{A}_{c}, \mathcal{A}_{r}$ and vector $\mathcal{F}_{c}$ were simply loaded as datafiles. This, combined with the solution of the tracking filter before state estimation, yielded an algorithm which was sufficiently fast for implementation. Current efforts are aimed toward the simultaneous approximation of the tracking and state estimator equations during implementation.

\section{Algorithm 4.1.2: Discrete Time Control of Periodic Excitation}

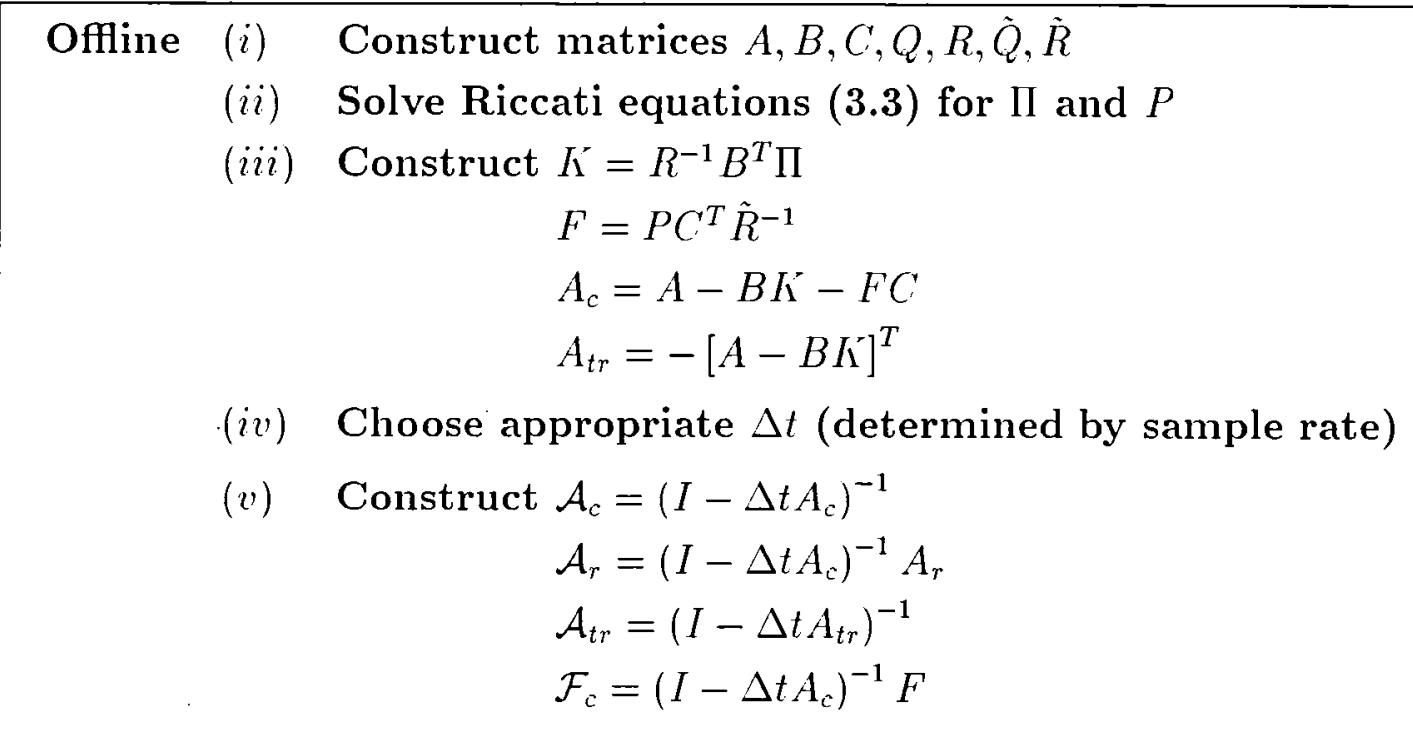

Online (i) Collect acceleration data $a\left(t_{j}\right)$

(ii) Integrate to obtain $y_{o b}\left(t_{j}\right)=v\left(t_{j}\right)$

(iii) Approximate and store tracking values

$$
\begin{aligned}
r_{j+1} & =\mathcal{A}_{t r} r_{j}+\mathcal{A}_{t r} \hat{g}\left(t_{j}\right) \\
r(\tau) & =0
\end{aligned}
$$

(iii) Time step the discrete estimator system

$$
\begin{aligned}
& y_{c_{j+1}}=\mathcal{A}_{c} y_{c_{j}}+\mathcal{F}_{c} y_{o b}\left(t_{j}\right)+\mathcal{A}_{r} r_{j}+\mathcal{A}_{c} g\left(t_{j}\right) \\
& y_{c}(k \tau)=0
\end{aligned}
$$

(iv) Calculate the voltage $u\left(t_{j}\right)=-K y_{c_{j}}+R^{-1} B^{T} r_{j}$

\subsubsection{Higher-Order Approximations}

In the previous discussion, a modified backward Euler method was used to discretize the state estimator and tracking equations. As indicated, by numerical simulations reported in [14] and experimental results in the next section, for small $\Delta t$, this provides sufficient accuracy to calculate an effective feedback voltage. If more accuracy is needed, a trapezoid rule or hybrid method of the nature discussed on page 225 of [26] can be used. These provide increased accuracy without adding complexity during implementation since the components $\mathcal{A}_{t r}, \mathcal{A}_{c}, \mathcal{A}_{r}$ and $\mathcal{F}_{c}$ can still be computed offline. 


\subsection{Example 1 - Matrix Construction}

The control discussion thus far has been general in the sense that it holds for general systems of the form

$$
\begin{array}{lll}
\dot{y}(t)=A y(t)+B u(t) & \text { or } & \\
y_{o b}(t)=C y(t)=A y(t)+B u(t)+g(t) & y_{o b}(t)=C y(t)
\end{array}
$$

as long as the pair $(A, B)$ is stabilizable and $(A, C)$ is detectable. Moreover, the cost functional matrices $Q, R$ and observation matrices $\tilde{Q}, \tilde{R}$ have been treated as general clesign criteria to be specified according to the application under consideration. In this example, we illustrate explicitly the matrices and filters used when implementing these control techniques for a vibrating circular plate.

We first note that $N=16$ modified cubic splines (see (2.3)) were sufficient for resolving the plate dynamics in the frequency range under consideration. Due to the axisymmetric excitation and response of the plate, the Fourier limit $M=0$ was used in all calculations. Hence a total of $\mathcal{N}=16$ basis functions were used which led to 32 coefficients in the vector $y$.

The formulation and sizes of all components in the control system for the circular plate are

\begin{tabular}{|c|c|c|}
\hline Component & Size & Comments \\
\hline$A=\left[\begin{array}{ccc}0 & I & \\
-\left(M^{\mathcal{N}}\right)^{-1} & K_{D}^{\mathcal{N}} & -\left(M^{\mathcal{N}}\right)^{-1} K_{c_{D}}^{\mathcal{N}}\end{array}\right]$ & $32 \times 32$ & $\begin{array}{l}\text { The elements comprising the } \\
16 \times 16 \text { matrices } M^{\mathcal{N}}, K_{D}^{\mathcal{N}} \text { and } \\
K_{C_{D}}^{-\mathcal{N}} \text { are summarized in }(2.4)\end{array}$ \\
\hline$B=\left[\begin{array}{cc}0 & \\
\left(M^{\mathcal{N}}\right)^{-1} & \tilde{B}^{\mathcal{N}}\end{array}\right]$ & $32 \times 1$ & $\begin{array}{l}\text { See }(2.4) \text { for the description of } \\
\tilde{B}^{\mathcal{N}}\end{array}$ \\
\hline$g(t)=\left[\begin{array}{c}0 \\
\hat{g}^{\mathcal{N}}(t)\end{array}\right]$ & $32 \times 1$ & $\begin{array}{l}\text { The elements of } \hat{g}^{\mathcal{N}}(t) \text { are de- } \\
\text { tailed in }(2.4)\end{array}$ \\
\hline$C=\left[\begin{array}{c|c}0, \cdots, 0 & B_{1}^{\mathcal{N}}\left(r_{1}, \theta_{1}\right), \cdots, B_{\mathcal{N}}^{\mathcal{N}}\left(r_{1}, \theta_{1}\right) \\
\vdots & \vdots \\
0, \cdots, 0 & B_{1}^{\mathcal{N}}\left(r_{p}, \theta_{p}\right), \cdots, B_{\mathcal{N}}^{\mathcal{N}}\left(r_{p}, \theta_{p}\right)\end{array}\right.$ & $p \times 32$ & $\begin{array}{l}\text { In the experiments, accelera- } \\
\text { tion data was integrated to } \\
\text { obtain velocity values which } \\
\text { are the second state values in } \\
\text { the second-order formulation. } \\
\text { Since one accelerometer was } \\
\text { used, } p=1 \text {. See Example } 2 \\
\text { of Section } 4.3 \text { for a discussion } \\
\text { regarding the duality between } \\
\text { control and observation. }\end{array}$ \\
\hline
\end{tabular}
summarized in Figure 4.2 and 4.3 below.

Figure 4.2. System and control matrices used in the circular plate experiments. 


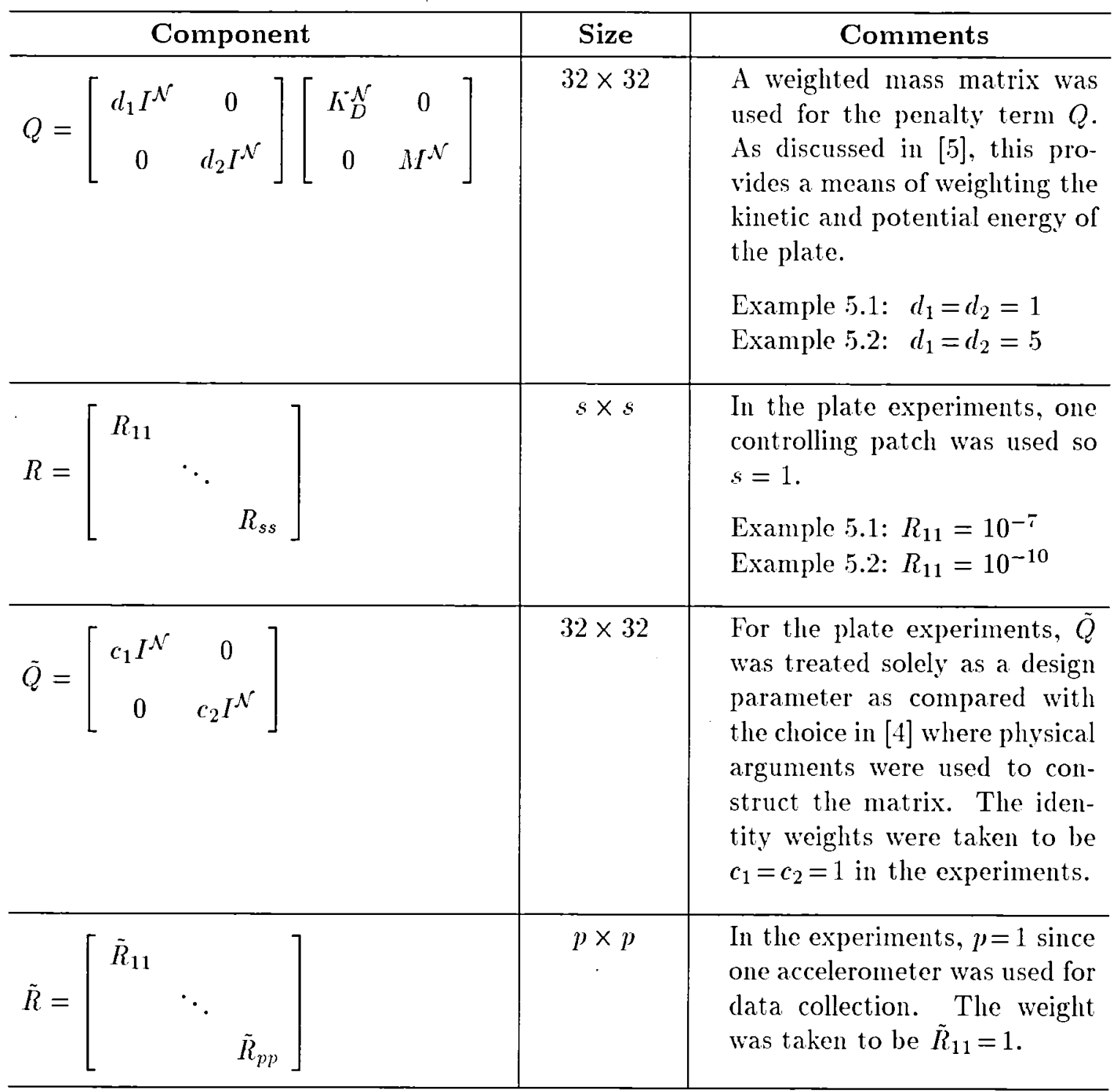

Figure 4.3. Control and observation matrices used in the circular plate experiments. The experimental results in Example 5.1 demonstrate control of transient dynamics while the periodic case is illustrated in Example 5.2.

These matrices and vectors are then employed in Algorithms 4.1.1 or 4.1.2 to create the implementation matrices and filters which were ultimately used in the experiments. 


\subsection{Example 2 - Duality Between Control and Observation}

The duality between control and observation can be noted by considering the form of the control matrix $B$ and observation matrix $C$. Illustrating with the case in which $s$ patch pairs are used for control, the $2 \mathcal{N} \times s$ control matrix has the form

$$
B=\left[\begin{array}{cc}
0 & \\
\left(M^{\mathcal{N}}\right)^{-1} & \tilde{B}^{\mathcal{N}}
\end{array}\right]
$$

where the $\mathcal{N} \times s$ matrix $\dot{B}^{\mathcal{N}}$ has elements

$$
\begin{aligned}
{\left[\dot{B}^{\mathcal{N}}\right]_{\ell, j} } & =\int_{\Gamma_{0}} \mathcal{K}_{j} \overline{\nabla^{2} B_{\ell}^{\mathcal{N}}} \lambda_{j}(r, 0) d \gamma \\
& =\int_{j^{\text {th }} \text { patch }} \mathcal{K}_{j} \overline{\nabla^{2} B_{\ell}^{\mathcal{N}}} d \gamma
\end{aligned}
$$

(here $\chi_{j}(r, \theta)$ denotes the characteristic function over the $j^{\text {th }}$ patch).

When data from accelerometers located at the points $\left(r_{1}, \theta_{1}\right), \cdots,\left(r_{p}, \theta_{p}\right)$ is integrated to obtain velocity values, the $p \times 2 \mathcal{N}$ observation matrix is given by

$$
C=\left[\begin{array}{ll}
0 & 1
\end{array}\right]\left[\begin{array}{cc}
\dot{C} & 0 \\
0 & \hat{C}
\end{array}\right]
$$

where

$$
\begin{aligned}
{\left[\tilde{C}_{j, \ell}\right.} & =\int_{\Gamma_{0}} B_{\ell}^{\mathcal{N}} \delta\left(r_{j}, \theta_{j}\right) d \gamma \\
& =B_{\ell}^{\mathcal{N}}\left(r_{j}, \theta_{j}\right) .
\end{aligned}
$$

With $C$ thus defined, it can immediately be noted that

$$
\begin{aligned}
y_{o b}(t) & =C y(t) \\
& =\sum_{k=1}^{\mathcal{N}} \dot{w}_{k}(t) B_{k}^{\mathcal{N}}\left(r_{j}, \theta_{j}\right)
\end{aligned}
$$

denotes the physical value of the velocity at the point $\left(r_{j}, \theta_{j}\right)$ given by the state equations at time $t$. This is an approximation, to within modeling and processing error, of the actual plate plate velocity $v(t)$ which is measured in experiments.

Similarly, multiplication of the state estimator coefficients $y_{c}$ by $C$ produces an estimate of the velocity which is then compared against the measured plate velocity when integrating the state estimator equation

$$
\dot{y}_{c}(t)=\left[A-B R^{-1} B^{T} \Pi\right] y_{c}(t)+P C^{T} \tilde{R}^{-1}\left[v(t)-C y_{c}(t)\right] .
$$

It should be noted that as the state estimates approach the measured plate values, the estimator equation approaches the state equation

$$
\dot{y}(t)=A y(t)-B R^{-1} B^{T} \Pi y_{c}(t)
$$

which is used to model the plate dynamics. 


\subsection{Integration of Experimental Data}

For the experiments involving the control of circular plate vibrations, data consisted of acceleration measurements obtained from one or more accelerometers on the plate. It was then necessary to approximately integrate this data to obtain velocity values so as to have a state variable for control calculations. An issue which turns out to be crucial when approximately integrating experimental data concerns the robustness of the integrator with respect to inexact initial conditions and DC gains or biases (added constants) in the data. The inexact initial conditions can be due to unknown system contributions, static shocks during system connections, et cetera. While careful calibration can alleviate some of the uncertainty in initial conditions, it cannot fully eliminate the problem. The problem of gains or biases due to small DC voltages in the system can also be minimized but never fully eliminated. Hence an integrator which is minimally affected by uncertain initial conditions and DC gains in the data is crucial for success when approximately integrating data.

Here we consider two techniques for approximately integrating acceleration data to obtain velocity values in accordance with the relation

$$
\dot{v}(t)=a(t) .
$$

Essentially, the idea is to replace the integration by either the first-order differential equation

$$
\dot{v}+\Omega v=\frac{1}{R C} a
$$

or the second-order equation

$$
\ddot{v}+\Omega \dot{v}+\Omega^{2} v=\frac{1}{R C} \dot{a}
$$

(see [21]). The design parameters $\Omega$ and $R C$ are frequency and time constants, respectively, which are chosen so that $R C=1$ and $\omega>6 \Omega$, where $\omega$ is the smallest observed frequency. For solution, (4.2) is written as the first-order system

$$
\begin{aligned}
& {\left[\begin{array}{l}
\dot{v} \\
\epsilon
\end{array}\right]=\left[\begin{array}{cc}
-\Omega & \Omega \\
-\Omega & 0
\end{array}\right]\left[\begin{array}{l}
v \\
\epsilon
\end{array}\right]+\left[\begin{array}{c}
\frac{a(t)}{R C} \\
0
\end{array}\right]} \\
& \Rightarrow \dot{z}=A z+f
\end{aligned}
$$

where $\dot{e}=-\Omega v$. The integration of (4.3) is subject to the initial conditions

$$
\left[\begin{array}{l}
v(0) \\
e(0)
\end{array}\right]=\left[\begin{array}{l}
v_{0} \\
\epsilon_{0}
\end{array}\right] .
$$

As will be detailed in the subsequent discussion, the first-order integrator (4.1) is robust with respect to inexact initial conditions but propagates DC gains in the data. In the secondorder integrator (4.2), or system (4.3), both disturbance in initial conditions and DC gains in acceleration data are exponentially attenuated. Moreover, the frequency response of this approximator is very close to that in the original signal for $\omega>6 \Omega$. Hence this latter method provides an accurate and robust means of approximately integrating experimental data. 


\subsubsection{First-Order Approximate Integrator}

Here we examine the properties of the first-order approximate integrator (4.1). We consider first the case in which the exact initial condition $v_{0}$ is known and the acceleration $a(t)$ is free from DC gains or biases (added constants). If we let $V(s)=\mathcal{L}\{v(t)\}$ and $A(s)=\mathcal{L}\{a(t)\}$, then Laplace transformation of the system (4.1) yields

$$
V(s)=\frac{1}{s+\Omega} v_{0}+\frac{1}{s+\Omega} \cdot \frac{1}{R C} A(s) .
$$

Hence the approximate integrator (4.1) is a single pole filter. Inverse transformation then yields

$$
v(t)=\epsilon^{-\Omega t} v_{0}+\frac{1}{R C} \int_{0}^{t} \epsilon^{-\Omega(t-s)} a(s) d s
$$

as the solution to $(4.1)$.

Similarly, if $\tilde{v}_{0}$ denotes a perturbed initial condition and a DC gain $\tilde{g}_{a}$ is present in the data, then the solution is given by

$$
\tilde{v}(t)=\epsilon^{-\Omega t} \tilde{v}_{0}+\frac{1}{R C} \int_{0}^{t} \epsilon^{-\Omega(t-s)}\left[a(s)+\tilde{g}_{a}\right] d s .
$$

It follows immediately that

$$
\tilde{v}(t)=v(t)+e^{-\Omega t}\left(\tilde{v}_{0}-v_{0}-\frac{\grave{g}_{a}}{R C \Omega}\right)+\frac{\check{g}_{a}}{R C \Omega} .
$$

It is first noted that the perturbations in initial conditions exponentially decay with the rate of decay influenced by the magnitude of the parameter $\Omega$. DC gains of the order $\hat{g}_{a} /(R C \Omega)$ remain, however, thus leading to difficulties when such biases are present in the data. Both properties are numerically illustrated through examples in [14].

The manner through which the solution (4.5) approximates the solution to the original relation $\dot{v}(t)=a(t)$ can be illustrated with a simple example. Consider $a(t)=120 \pi \cos (120 \pi t)$. The solution to (4.1) for this acceleration is

$$
v(t)=\frac{1}{R C} \cdot \frac{120 \pi}{\Omega^{2}+(120 \pi)^{2}}[\Omega \cos (120 \pi t)+120 \pi \sin (120 \pi t)]-\frac{1}{R C} \cdot \frac{120 \pi \Omega}{\Omega^{2}+(120 \pi)^{2}} e^{-\Omega t}
$$

which reduces to the solution of the original relation with $\Omega=0$ and $R C=1$. For $\Omega=16 \pi$, the solution $v$ still provides an adequate approximation to the original whereas it is a very poor approximation with $\Omega=120 \pi$. This phenomenon is illustrated in [14].

\subsubsection{Second-Order Approximate Integrator}

The second-order approximate integrator (4.2), or equivalent system (4.3), eliminates the difficulties associated with both inexact initial conditions and DC gains or biases in the data. The elimination of constants in the data can heuristically be attributed to the differentiation of the acceleration data. This can be made rigorous by analytically solving the problem. 
We again let $V(s)=\mathcal{L}\{v(t)\}$ and $A(s)=\mathcal{L}\{a(t)\}$ and let $v_{0}, v_{1}$ and $a_{0}$ denote initial conditions. Transformation of $(4.2)$ yields

$$
\left[s^{2} V(s)-s v_{0}-v_{1}\right]+\Omega\left[s V(s)-v_{0}\right]+\Omega^{2} V(s)=\frac{1}{R C}\left[s A(s)-a_{0}\right]
$$

from which it follows that

$$
V(s)=\frac{s+\Omega}{s^{2}+\Omega s+\Omega^{2}} v_{0}+\frac{\left(v_{1}-a_{0} / R C\right)}{s^{2}+\Omega s+\Omega^{2}}+\frac{s}{s^{2}+\Omega s+\Omega^{2}} \cdot \frac{1}{R C} A(s) .
$$

Inverse transformation then yields the solution

$$
\begin{aligned}
v(t) & =e^{-\Omega t / 2}\left[\cos (\sqrt{3} \Omega t / 2) v_{0}+\frac{1}{\sqrt{3}} \sin (\sqrt{3} \Omega t / 2) v_{0}+\frac{2}{\sqrt{3} \Omega} \sin (\sqrt{3} \Omega t / 2)\left(v_{1}-\frac{a_{0}}{R C}\right)\right] \\
& +\frac{1}{R C} \int_{0}^{t} \epsilon^{-\Omega(t-s) / 2}\left[\cos (\sqrt{3} \Omega(t-s) / 2)-\frac{1}{\sqrt{3}} \sin (\sqrt{3} \Omega(t-s) / 2)\right] A(s) d s .
\end{aligned}
$$

As in the discussion of (4.1), we then consider the corresponding solution with perturbed initial conditions $\grave{v}_{0}, \tilde{v}_{1}$ and $\grave{a}_{0}$ and DC gain $\grave{g}_{a}$. In this case, the perturbed solution $\check{v}(t)$ is given by

$$
\begin{aligned}
\dot{v}(t) & =v(t)+\epsilon^{-\Omega t / 2}\left[\cos (\sqrt{3} \Omega t / 2)+\frac{1}{\sqrt{3}} \sin (\sqrt{3} \Omega t / 2)\right]\left[\tilde{v}_{0}-v_{0}\right] \\
& +\epsilon^{-\Omega t / 2} \cdot \frac{2}{\sqrt{3} \Omega} \sin (\sqrt{3} \Omega t / 2)\left[\left(\hat{v}_{1}-\frac{\tilde{a}_{0}}{R C}\right)-\left(v_{1}-\frac{a_{0}}{R C}\right)\right] \\
& +\epsilon^{-\Omega t / 2} \cdot \frac{2 \tilde{g}_{a}}{\sqrt{3} R C \Omega} .
\end{aligned}
$$

Here both the perturbations in initial conditions and the added constants in the data exponentially decay with the rate of decay dependent on the magnitude of $\Omega$. This is illustrated in examples given in [14]. We reiterate that while increased values of $\Omega$ lead to more rapid decay of perturbations and biases, the solution to the differential equation less accurately approximates the true velocity. This, in combination with the goal of accurately preserving signal frequencies, leads to the condition $\Omega<\omega / 6$.

\subsubsection{Numerical Approximation}

Because of the potential for problems involving DC gains with the first-order formulation (4.1), we concentrate primarily on the second-order filter (4.2) and the corresponding system (4.3). We note that similar scalar techniques can be used to approximate the solution to (4.1). In considering numerical techniques for integrating (4.3), emphasis was placed on using a technique which could easily be implemented in real time. The two methods considered here are Euler's method and a backward Euler's method. The two are summarized below.

\section{Euler's Method:}

$$
\begin{aligned}
& z\left(t_{k+1}\right)=[I+d t A] z\left(t_{k}\right)+d t f\left(t_{k}\right) \\
& \Rightarrow\left[\begin{array}{l}
v \\
\epsilon
\end{array}\right]\left(t_{k+1}\right)=\left[\begin{array}{cc}
1-d t \Omega & d t \Omega \\
-d t \Omega & 1
\end{array}\right]\left[\begin{array}{l}
v \\
\epsilon
\end{array}\right]\left(t_{k}\right)+\left[\begin{array}{c}
d t \frac{a\left(t_{k}\right)}{R C} \\
0
\end{array}\right]
\end{aligned}
$$




\section{Backward Euler's Method:}

$$
z\left(t_{k}\right)=[I-d t A]^{-1} z\left(t_{k-1}\right)+[I-d t A]^{-1} d t f\left(t_{k-1}\right)
$$

where

$$
\begin{aligned}
& {[I-d t A]^{-1}=\left[\begin{array}{cc}
\frac{1}{1+d t \Omega+(d t \Omega)^{2}} & \frac{d t \Omega}{1+d t \Omega+(d t \Omega)^{2}} \\
\frac{-d t \Omega}{1+d t \Omega+(d t \Omega)^{2}} & \frac{1+d t}{1+d t \Omega+(d t \Omega)^{2}}
\end{array}\right]} \\
& d t[I-d t A]^{-1} f\left(t_{k-1}\right)=\left[\begin{array}{c}
\frac{(d t / R C) a\left(t_{k-1}\right)}{1+d t \Omega+(d t \Omega)^{2}} \\
\frac{\left(-d t^{2} \Omega / R C\right) a\left(t_{k-1}\right)}{1+d t \Omega+(d t \Omega)^{2}}
\end{array}\right] .
\end{aligned}
$$

The advantage of backward Euler's method over Euler's method is its stability properties with slightly more involved matrices being the disadvantage. Numerical examples demonstrating both methods with a variety of exogenous forces can be found in [14].

\section{$5 \quad$ Experimental Results}

Experimental results demonstrating both the transient and steady state capabilities of the control methodology are presented in this section. The circular plate used in these experiments had a radius of $9^{\prime \prime}(.2276 \mathrm{~m})$ and a thickness of $.05^{\prime \prime}(.00127 \mathrm{~m})$. A pair of piezoceramic patches having radius $.75^{\prime \prime}(.01905 \mathrm{~m})$ and thickness $.007^{\prime \prime}(.0001778 \mathrm{~m})$ were bonded to the center of the plate. In both the transient and steady state experiments, only one patch was used for control. In the steady state experiments, the opposite patch was used to drive the plate while it was allowed to remain uncharged in the transient case. The plate was mounted in a wooden frame by a circular aluminum collar which provided boundary conditions which were sufficiently close to clamped (zero displacement and slope).

The first step in the process was the estimation of physical parameters through fit-to-data techniques. As detailed in $[2,3]$, transient plate vibrations were excited though an impact hammer strike or the input of a voltage spike to the patches, and acceleration data was measured. The parameter values summarized in Table 5.1 were obtained through a least squares minimization of the difference between the model response and the measured data. These values were then employed when constructing the component matrices $A, B$ and $Q$ (see Figure 4.2) used during the experimental implementation of the controller. 


\begin{tabular}{c|c|c}
\hline \multicolumn{2}{c|}{} & Physical Parameters \\
\hline $\begin{array}{c}\rho \cdot h \\
\left(\mathrm{~kg} / \mathrm{m}^{2}\right)\end{array}$ & Plate & 3.170 \\
\hline$D$ & Plate + Pzt & 3.216 \\
$(N \cdot m)$ & Plate + Pzt & 11.151 \\
\hline$c_{D}$ & Plate & 11.506 \\
$(N \cdot m \cdot s e c)$ & Plate + Pzt & $1.443-4$ \\
\hline$\nu$ & Plate & .326 \\
\hline \multicolumn{2}{c}{$\gamma\left(s \in c \cdot N / m^{3}\right)$} & .325 \\
\hline $\mathcal{K}^{B}(N / V)-$ Controlling Patch & 17.021 \\
\hline \multicolumn{2}{c|}{$\mathcal{K}^{B}(N / V)-$ Driving Patch } & .016 \\
\hline
\end{tabular}

Table 5.1. Physical parameters used in the experiments.

\subsection{Transient Control}

To investigate the capabilities of the method for controlling transient vibrations, decaying plate responses generated by an impact hammer strike were considered. In each case, the strike was directed to the center of the plate and hence the plate response was axisymmetric. In the first set of experiments, data was collected from an accelerometer located at the plate center on the side opposite from the hammer impact; thus $P_{1}=\left(r_{1}, \theta_{1}\right)=(0,0)$ in the construction of the observation matrix $C$ described in Figure 4.2. The experiments were then repeated with the accelerometer placed at the off-center point $P_{2}=\left(r_{1}, \theta_{1}\right)=\left(2^{\prime \prime}, 0\right)$ to illustrate that collocation between the sensor and actuator is unnecessary in this control method (see Figure 5.1 for sensor, actuator and impact locations). The results obtained with the off-center accelerometer are presented here, and the reader is referred to [14] for a discussion of the transient control results obtained with observations from the centered accelerometer.
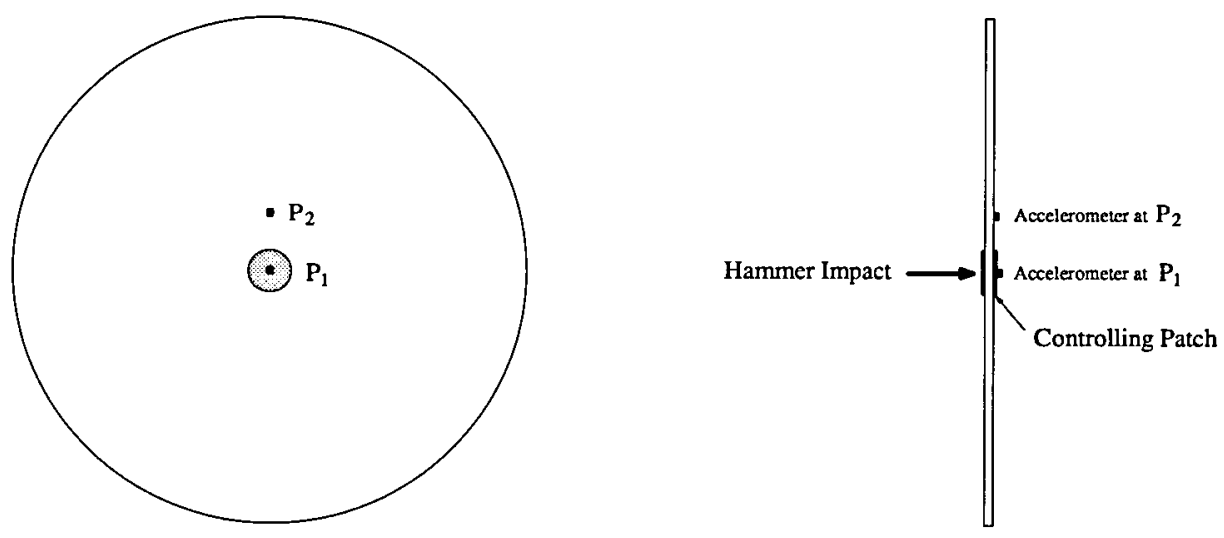

Figure 5.1. Patch, accelerometer and impact locations for the experiments involving control of transient vibrations. 
Since no exogenous force was applied to the plate, the state estimator and control law summarized in Algorithm 4.1.1 were used to compute the controlling voltage to the patch. The component matrices as well as the cost functional and observation parameters used in these experiments are summarized in Figures 4.2 and 4.3.

Data acquisition and processing were performed with a PC-based Texas Instruments TMS 320-C.30 digital signal processing (DSP) board. A schematic of the amplifiers, filters, DSP configuration and $\mathrm{PC}$ algorithm is given Figure 5.2. In the experiments, the accelerometer voltage was initially boosted by a factor of 10 and then reduced by $5 \mathrm{~dB}$ before reaching the DSP board. The controlling voltage output from the DSP was also boosted by an amplifier before input to the patches. This was necessary since the maximum voltage output by the DSP is $2.5 \mathrm{~V}$ whereas $60-70 \mathrm{~V}$ were needed at the patch. The reader is also referred to Figure 4.1 for an illustration of the experimental process and to [14] for details regarding the implementation process. We point out that the ratio $\frac{2048}{2.5 \mathrm{~V}}$, illustrated in the A/D conversion, results when the voltage range -2.5 to 2.5 is discretized into 4096 possible digital values. The reciprocal process occurs when digital values are converted to analog voltages in the $D / A$ converter.

The control code was written in assembler in order to attain sufficiently fast sample rates for resolving transient frequencies excited by the hammer impact. While the code ran at rates greater than $7 \mathrm{KHz}$, a sample rate of $3.5 \mathrm{KHz}$ was used in the experiments. This proved to be sufficient for resolving the three axisymmetric modes (with frequencies of $60 \mathrm{~Hz}, 227 \mathrm{~Hz}$ and $512 \mathrm{~Hz}$ ) excited in the experiments.

Representative plots of the plate velocity (integrated from the data) at the off-center point $P_{2}$ in the uncontrolled and controlled cases are given in Figure 5.3 with reduction levels at times $t=0.5,1.0,1.5 \mathrm{sec}$ summarized in Table 5.2. The percentage reductions were calculated by determining the ratio between the maximum values of the controlled and uncontrolled trajectories through one period containing the time point of interest. As illustrated by the results in the table and figure, the velocity level in the controlled case has been reduced by $50 \%$ before $.5 \mathrm{~s} \in \mathrm{c}$ and is essentially fully attenuated by $1.5 s \in c$. We reiterate that these results were obtained with data obtained from an accelerometer at $\left(2^{\prime \prime}, 0\right)$ and a centered actuating patch thus illustrating that collocation is unnecessary for this control method.

The voltage, $u\left(t_{j}\right)=-K y_{c_{j}}$, was recorded in each experiment and that yielding the control results reported here is plotted in Figure 5.4. It is noted that the voltage has a maximum magnitude of $70 \mathrm{~V}$. In practice, it has been observed that the patches can be used for extended periods at the frequencies of interest without damage or degradation of performance if the voltage levels are maintained below $\delta-10 \mathrm{rms} V /$ mil [2S]. Hence control voltage levels required for control of the transient vibrations is well within the tolerance of the $T$ mil patches used in the experiments.

Finally, the force delivered by the hammer impact in the uncontrolled and controlled cases is plotted in Figure 5.5 so as to provide a means of testing the equity of excitation levels in the uncontrolled and controlled experiments (the initial velocities in the two cases can also be compared to determine whether the same level of energy is being delivered in each experiment). As indicated by the results in this latter figure, the force delivered in the two cases is nearly identical (a slight double hit was always present when an impact hammer was used to excite the axisymmetric modes). 


\begin{tabular}{c|cc}
\hline Time & Acceleration & Velocity \\
\hline $.5 \mathrm{sec}$ & 69.5 & 68.2 \\
$1 \mathrm{sec}$ & 88.9 & 84.7 \\
$1.5 \mathrm{sec}$ & 93.8 & 97.8 \\
\hline
\end{tabular}

Table 5.2. The percent reductions in acceleration and velocity levels at the point $P_{2}=\left(2^{\prime \prime}, 0\right)$ when feedback control is implemented.

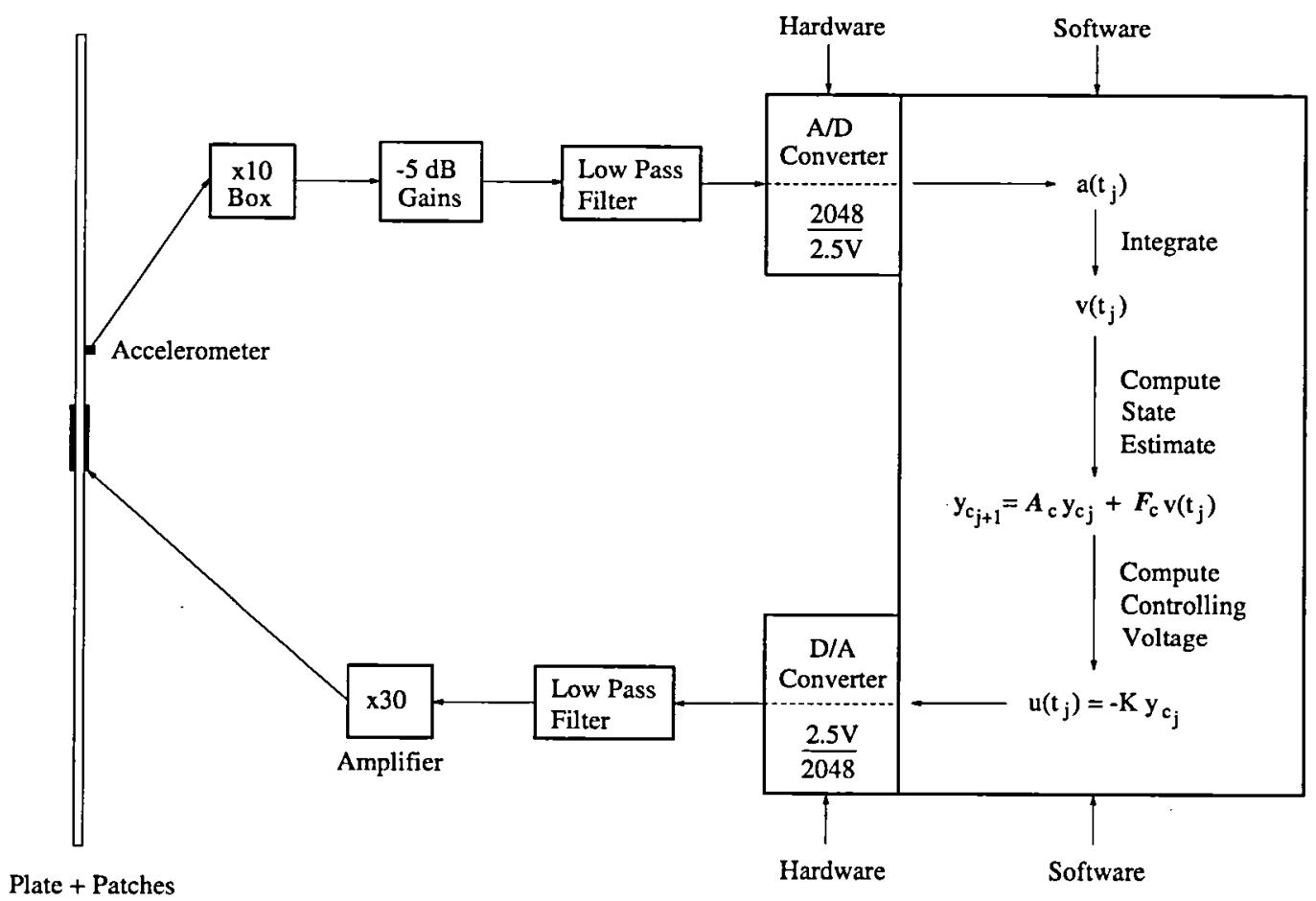

Figure 5.2: Amplifiers, DSP configuration and PC Algorithm 4.1.1 for controlling a plate excited by an initial impact. Component matrices are defined in Figures 4.2 and 4.3 . 


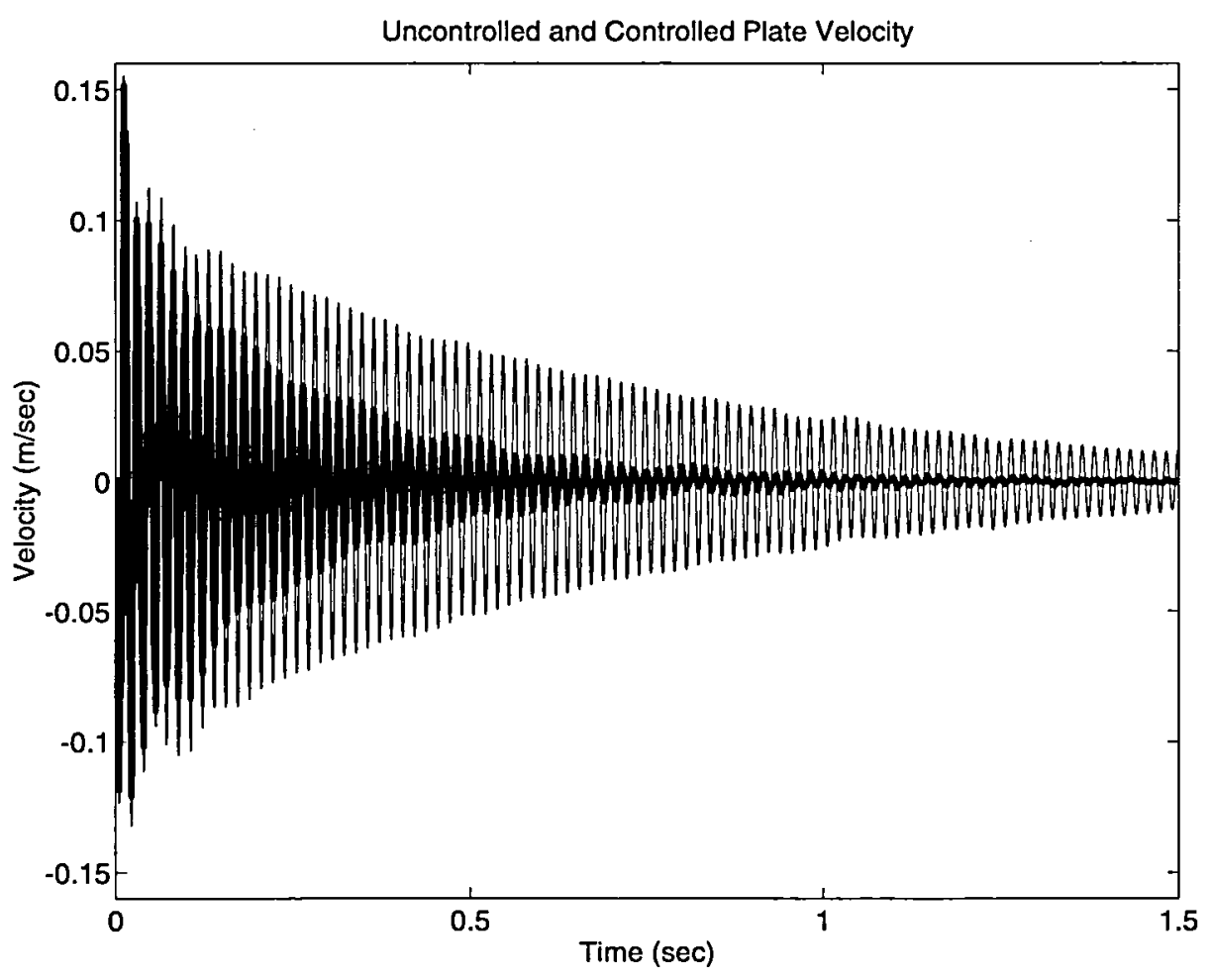

Figure 5.3. Uncontrolled and controlled plate vibrations at $\left(2^{\prime \prime}, 0\right)$ in response to an impact hammer hit; - (Uncontrolled), - (Controlled).

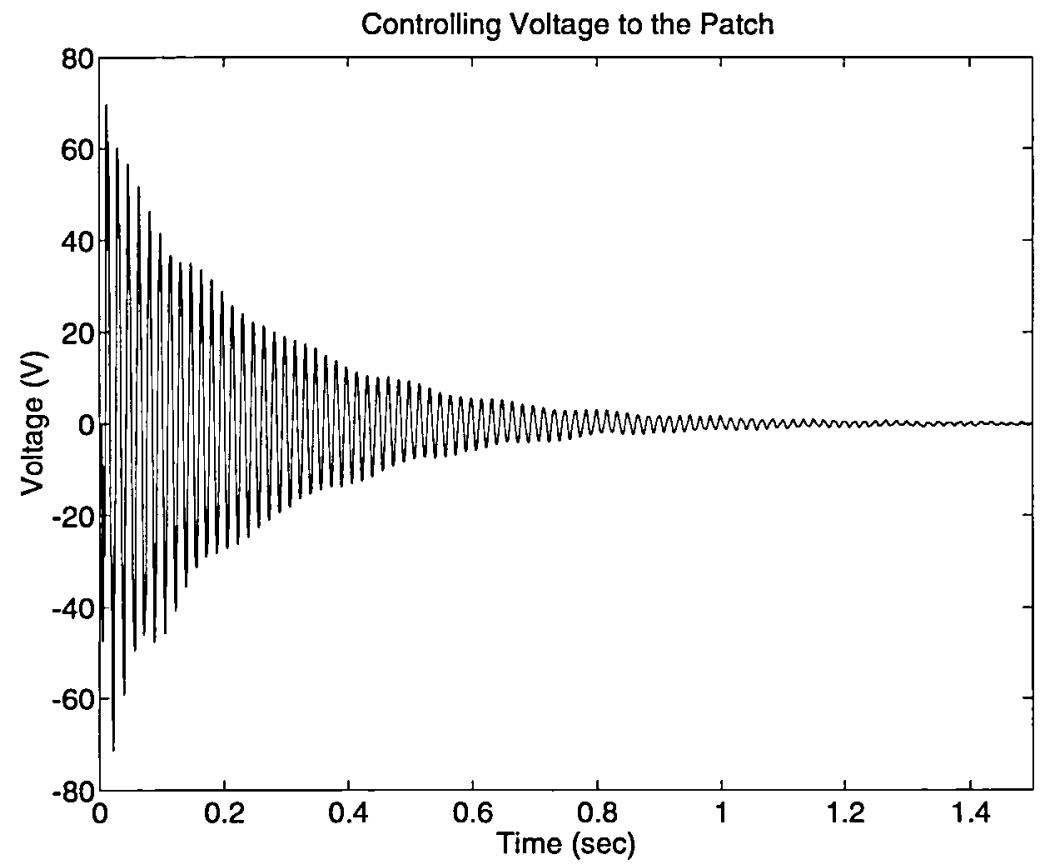

Figure 5.4. The controlling voltage for acceleration data observed at $\left(2^{\prime \prime}, 0\right)$. 


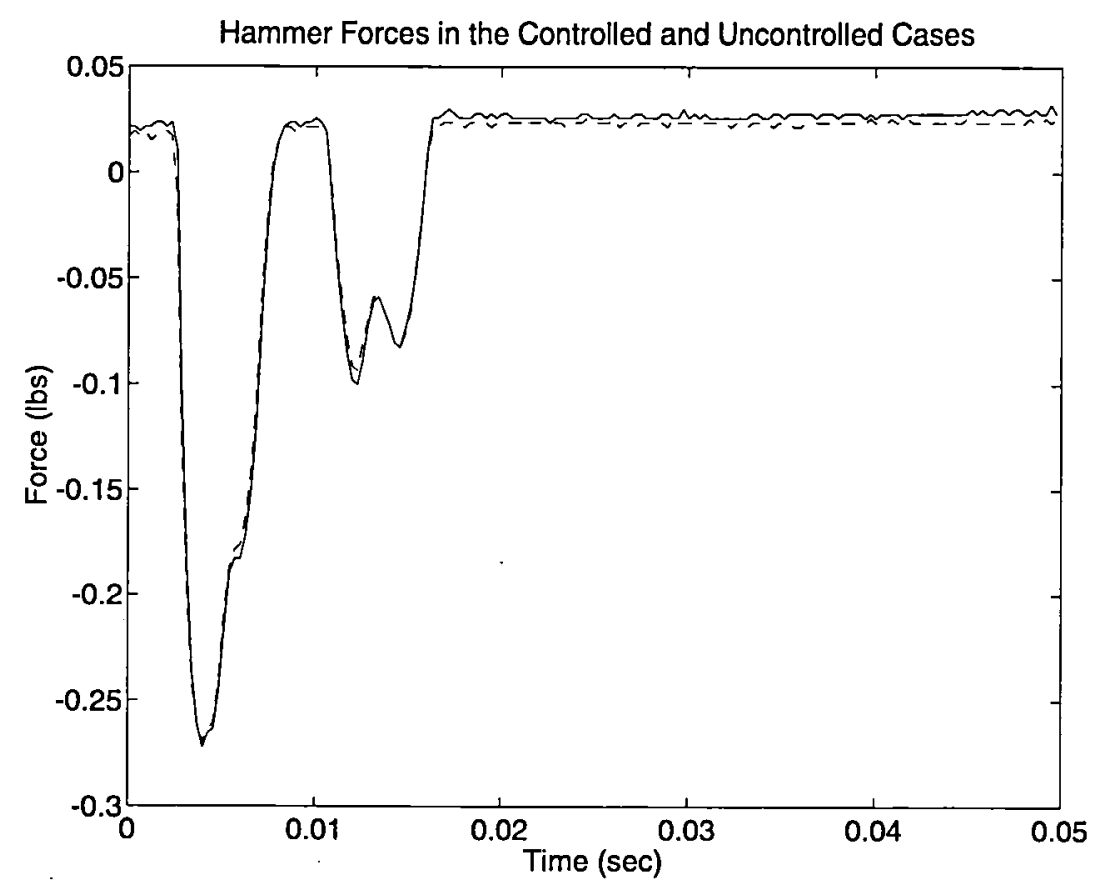

Figure 5.5. The force delivered by the impact hammer in the uncontrolled and controlled cases, —_ (Controlled Case), - - - (Uncontrolled Case).

\subsection{Control of a Periodic Exogenous Force}

A second problem under consideration concerns the control of plate vibrations when the plate is driven by a periodic exogenous force. To demonstrate the control capabilities in this case, a periodic driving voltage was supplied to one centered patch on the plate and the patch on the opposite side of the plate was used as the control actuator. Experimental tests indicated that a $350 \mathrm{~Hz}$ driving voltage produced a strong plate response and all tests were conducted with the exogenous voltage at that frequency.

Two sources were used to generate this exogenous signal: namely, an external oscillator and the $\mathrm{PC}$ running the control algorithm. As reported under Case 1 below, a purely steady state response could be considered with the oscillator-generated exogenous force since the plate was driven to steady state before the control program was initiated. Both a transient and steady state response were noted in the PC-generated signal since the input of the exogenous force to the plate began at the same time that the control algorithm was started. This latter means of excitation is considered in Case 2.

Since the system was driven by a periodic exogenous force, the discrete time Algorithm 4.1.2 (corresponding to continuous time Algorithm 3.3.1) was used to calculate the controlling voltage to the actuating patch. Again, component matrices as well as control and observation parameters are summarized in Figures 4.2 and 4.3.

For the off-center results reported here, velocity observation values were obtained by integrating data from an off-center accelerometer located at the point $P_{2}=\left(2^{\prime \prime}, 0\right)$ as depicted in 
Figure 5.1. The second input to the algorithm consisted of measurements $g\left(t_{j}\right)$ of the force driving the plate. We point out that when implementing this control method, both phase and magnitude information for the driving force were required as compared with many other methods (e.g., feedforward) which require only phase information.

In these experiments, the tracking components $r_{j}$ were calculated first and stored in a circular buffer. These values were then used when calculating the state estimates $y_{j}$ and voltages $u\left(t_{j}\right)$. While the implementation in this manner facilitated running the algorithm with sample rates on the order of $7 \mathrm{KHz}$ (again, the algorithm was coded in assembler), it limits the robustness of the method with respect to changes and variations in the driving force. Current efforts are directed toward simultaneous solution of the tracking and estimator difference equations.

A crucial issue when implementing the method concerns the handling of delays and phase shifts produced by the filters, $A / D$ and $D / A$ conversions, computation of the control voltages, et cetera. While the amount of delay and phase shift is frequency dependent, experiments indicated that at $350 \mathrm{~Hz}, 30-40^{\circ}$ phase shifts were introduced by the hardware. This was sufficient to destabilize the controller if left uncompensated. In the experiments, we compensated by first conducting an offline, numerical 'identification' to determine the amount of added delay necessary for stabilizing the controller in the presence of phase shifts of the order introduced by the experimental hardware. A summary of these results can be found in [14]. This is analogous to the online tuning or phase locking which is necessary for ensuring stability in other control methods. The numerical tests, summarized in [14], indicated that the introduction of a $216^{\circ}$ delay in accelerometer or exogenous force data would stabilize the systems, and this was implemented in the experiments by using a DAC delay box. The compensation for phase delays in this manner provides merely a first step toward optimal implementation of the method and one aspect of current research efforts is directed toward online compensation in the algorithm.

Data acquisition and processing was again performed with a $\mathrm{PC}$-based Texas Instruments TMS 320-C:30 board. A schematic of the setup is given in Figure 5.6. 


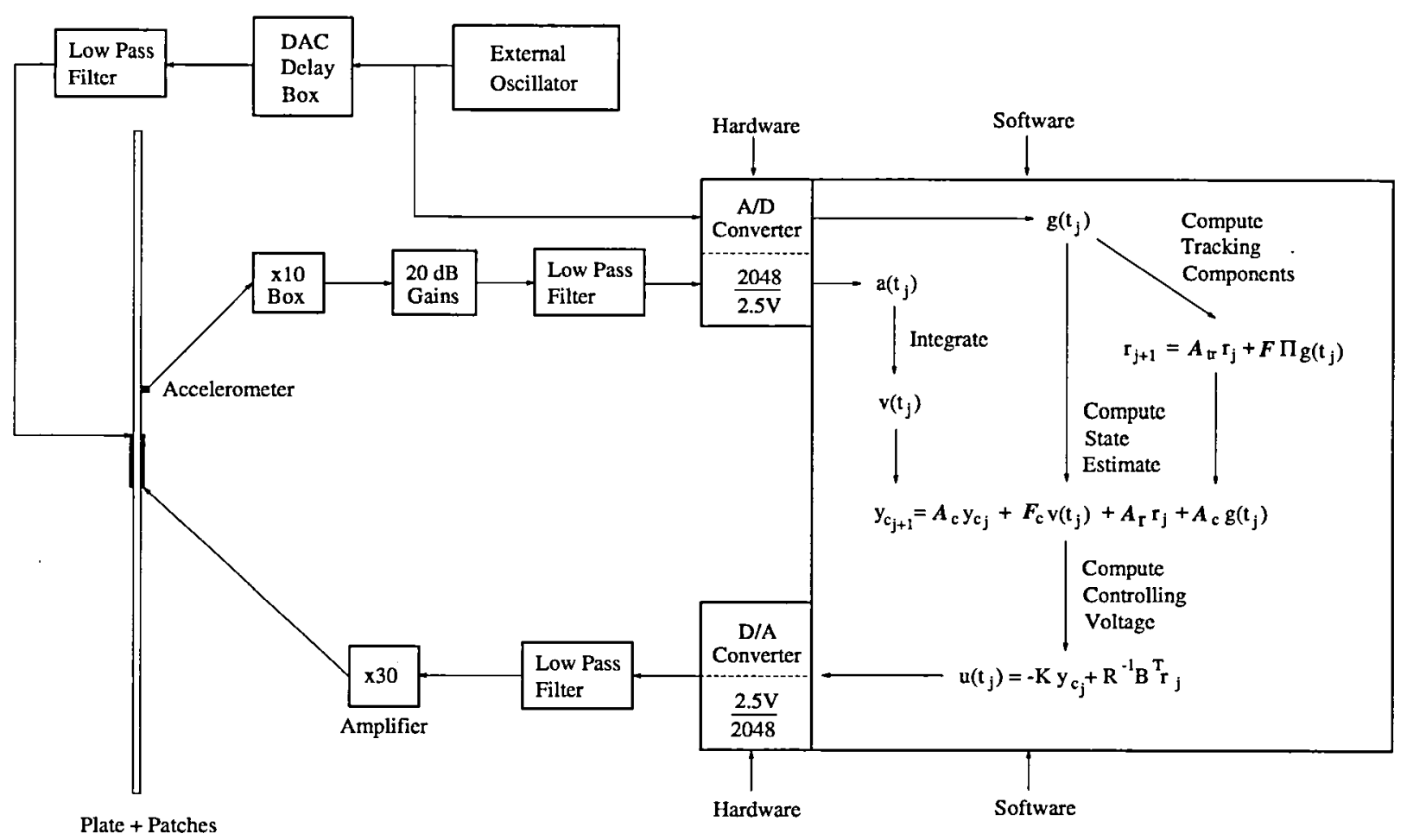

Figure 5.6. Amplifiers, DSP configuration and PC Algorithm 4.1.2 for controlling a plate driven by a periodic exogenous force. Component matrices are defined in Figures 4.2 and 4.3.

\section{Case 1: Oscillator-Generated Driving Signal}

For the results described here, the signal to the driving patch was generated by an external oscillator. The plate was allowed to reach steady state and then the control program was initiated. Acceleration levels measured by the accelerometer located at $P_{2}=\left(2^{\prime \prime}, 0\right)$ and integrated velocity values for the uncontrolled and controlled cases are plotted in Figure 5.7. As noted from the controlled trajectories in that figure, it takes the algorithm approximately 0.06 seconds to calculate and store a sufficient number of tracking components $r_{j}$. During that time interval, no voltage is fed to the actuating patch. Once the tracking calculations are completed, state estimation begins and the controlling voltage is computed and fed back into the system. The vibration levels decay for approximately 0.3 seconds and then are maintained at levels that are approximately $15 \%$ of those for the for the uncontrolled case for the remainder of the time interval. This corresponds to a $20 \log \left(a_{c o n} / a_{\text {uncon }}\right) \approx-16.5 \mathrm{~dB}$ reduction in acceleration levels.

While the magnitude of the controlling voltage is dependent upon the amplitude of the driving signal, magnitudes less than $40 \mathrm{~V}(28.3 \mathrm{~V} \mathrm{rms})$ were required to attain the levels reported here. At $350 \mathrm{~Hz}$, this was well within the range $(56-70 \mathrm{~V} \mathrm{rms}$ in this case) that was considered to be safe for the patch being used (see Example 5.1 for further discussion regarding the voltage levels at which the patches can be driven without damage or degradation in performance).

The results in this experiment demonstrate the effectiveness of the algorithm for controlling a system that has reached steady state. Hence one is not required to start the control with a system at rest. 

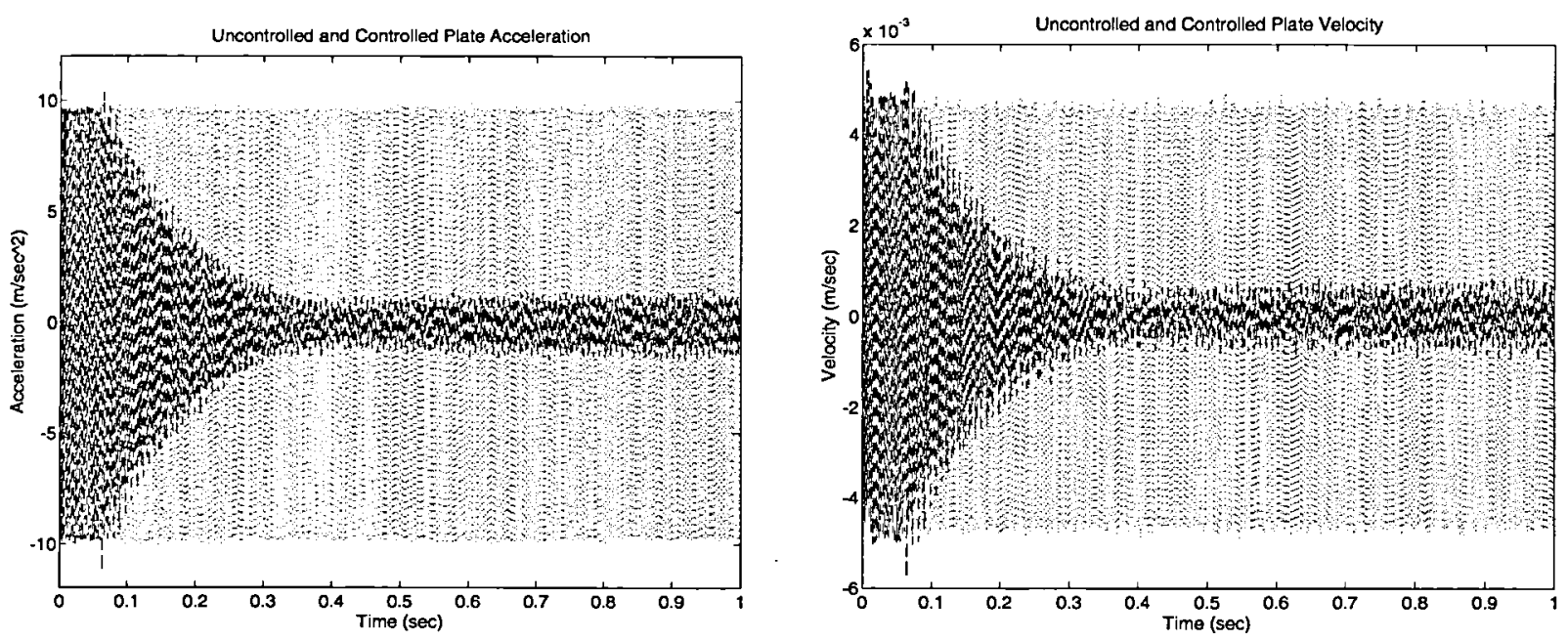

Figure 5.7. Uncontrolled and controlled plate acceleration and velocity at $(2,0)$ for system driven by periodic exogenous force; — (Uncontrolled), - (Controlled).

\section{Case 2: PC-Generated Driving Signal}

A second mechanism for generating the driving signal is with the PC that is used to process data and run the control algorithm. Acceleration and velocity plots of the uncontrolled and controlled plate vibrations excited in this manner are given in Figure 5.8. It can be seen that in this case, the plate starts from rest and is still being driven through a transient stage when the tracking calculations are completed and control begins. At that point, the controlled trajectories are reduced to the levels noted in the purely steady state case whereas the uncontrolled trajectories are driven to steady state. Here, an $82 \%(15 \mathrm{~dB})$ reduction in levels is noted at time $T=1$ second. This was obtained with a controlling voltage of magnitude $12 V_{\max }\left(8.5 V_{r m s}\right)$. These results demonstrate the effectiveness of the control algorithm for a system undergoing transient oscillations before reaching steady state in response to a periodic driving force.
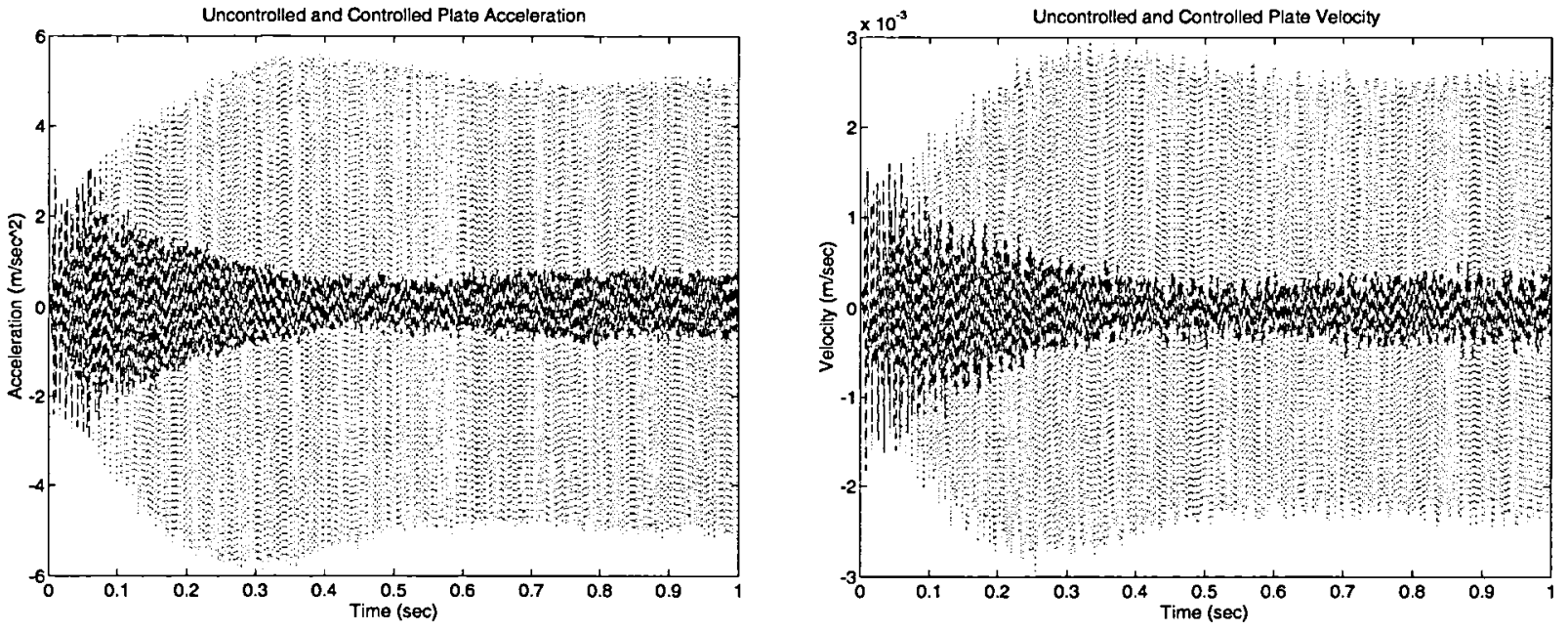

Figure 5.8. Uncontrolled and controlled plate acceleration and velocity at $(2,0)$ for system driven by periodic exogenous force; — (Uncontrolled), - (Controlled). 


\section{Conclusions}

In this work, the experimental implementation of a PDE-based controller was considered. While the motivating application involves the control of vibration levels for a circular plate through the excitation of surface-mounted piezoceramic patches, the general techniques described here will extend to a variety of applications.

For such control techniques, the first step is the derivation of a PDE model which accurately describes the dynamics of the system under consideration. Plysical parameters in these models must typically be estimated through fit-to-data techniques before control applications can be considered. Following a brief discussion regarding the strong and weak forms of a thin plate model with a discontinuous control input term (due to the piecewise constant nature of the piezoceramic patches), continuous-time LQG and $H^{\infty}$ methods for systems with no exogenous force or a periodic exogenous force were discussed. The discrete-time approximations necessary for implementing the methods with digital measurements were also presented. A crucial step when implementing the discrete-time controllers involves the approximate integration of data (e.g., accelerometer data integrated to obtain velocity state values), and first and second-order filters for accomplishing this were discussed. Without such filters, DC biases, that are always present in the data, would render the integrated values useless.

Experimental results demonstrating the control of transient and steady state vibrations were then presented. One advantage of the PDE-based controllers over standard frequency response input/output techniques is the capability for direct control of transient responses, and this was demonstrated in the first set of examples. A centered hammer impact was used to excite the plate and integrated data from an off-center accelerometer was used to reconstruct the state. The results demonstrate that attenuation levels on the order of $95 \%$ reduction can be attained by 1.5 seconds using the PDE-based controller.

The second example demonstrates the control of transient and steady state responses when the plate was driven by a periodic exogenous voltage to a secondary piezoceramic patch. These results demonstrate that, after accounting for hardware delays, attenuation levels on the order of $85 \%$ were attained when control was implemented. While implementation techniques are still being refined, these results demonstrate the effectiveness of the PDE-based controller for this system and indicate the potential of these control techniques for reducing transient and steady state dynamics in other structural and structural acoustic systems.

ACKNOWLEDGEMENTS: The authors extend their sincere thanks to Yun Wang, Brooks Air Force Base, for substantial collaboration throughout the duration of this work. 


\section{References}

[1] T. Bailey and J.E. Hubbard Jr., "Distributed Piezoelectric-Polymer Active Vibration Control of a Cantilever Beam," Journal of Guidance, 8(5), Sept. 1985, pp. 605-611.

[2] H.T. Banks, D.E. Brown, V. Metcalf, R.J. Silcox, R.C. Smith and Y. Wang, "A PDEBased Methodology for Modeling, Parameter Estimation and Feedback Control in Structural and Structural Acoustic Systems," Proceedings of the North American Conf. on Smart Structures and Materials, Orlando, FL, 1994, pp. 311-320.

[3] H.T. Banks, D.E. Brown, V. Metcalf, R.J. Silcox, R.C. Smith and Y. Wang, "The Estimation of Material and Boundary Parameters in a PDE-Based Circular Plate Model," to be submitted to Journal of Sound and Vibration.

[4] H.T. Banks, M.A. Demetriou and R.C. Smith "An $H^{\infty} /$ MinMax Periodic Control in a 2-D Structural Acoustic Model with Piezoceramic Actuators," Center for Research in Scientific Computation Technical Report, CRSC-TR94-9, June 1994, submitted to IEEE Trans. Auto Control.

[5] H.T. Banks, W. Fang, R.J. Silcox and R.C. Smith, "Approximation Methods for Control of Acoustic/Structure Models with Piezoceramic Actuators," Journal of Intelligent Material Systcms and Structures, 4(1), 1993, pp. 98-116.

[6] H.T. Banks and K. Ito, "Approximation in LQR Problems for Infinite Dimensional Systems with Unbounded Input Operators," Center for Research in Scientific Computation Technical Report, CRSC-TR94-22, November 1994, submitted to Journal of Mathematical Systems, Estimation and Control.

[7] H.T. Banks, K. Ito and B.B. King, "Theoretical and Computational Aspects of Feedback in Structural Systems with Piezoceramic Controllers," Computation and Control III, Proceedings of the Third Bozeman Conference, Bozeman, MT, 1992, Progress in Systems and Control Theory, Vol. 15, Birkhäuser Boston, Inc., 1993, pp. 1-27.

[\$] H.T. Banks, K. Ito and Y. Wang, "Computational Methods for Identification and Feedback Control in Structures with Piezoceramic Actuators and Sensors," Proceedings of the Conference on Recent Advances in Adaptive and Sensory Materials and their Applications, VPI and SU, Blacksburg, VA, April 1992, pp. 111-119.

[9] H.T. Banks and R.C. Smith, "The Modeling and Approximation of a Structural Acoustics Problem in a Hard-Walled Cylindrical Domain," Center for Research in Scientific Computation Technical Report, CRSC-TR94-26, December 1994.

[10] H.T. Banks and R.C. Smith, "Noise Control in a 3-D Structural Acoustic System: Numerical Simulations," Proceedings of the Second International Conference on Intelligent Materials, Williamsburg, VA, June 1994, 128-139. 
[11] H.T. Banks, R.C. Smith and Y. Wang, "Modeling Aspects for Piezoceramic Patch Activation of Shells, Plates and Beams," Center for Research in Scientific Computation Technical Report, CRSC-TR92-12, N.C. State University, to appear in Quarterly of Applied Mathematics.

[12] H.T. Banks, R.C. Smith and Y. Wang, "Vibration Suppression with Approximate Finite Dimensional Compensators for Distributed Systems: Computational Methods and Experimental Results," Proc. of the Second International Conference on Intelligent Materials, Williamsburg, VA, June 1994, 140-154.

[13] H.T. Banks, R.C. Smith and Y. Wang, "Modeling and Parameter Estimation for an Imperfectly Clamped Plate," to appear in Computation and Control IV. Proc. Fourth Bozeman Conf., Bozeman, MT, 1994, Progress in Systems and Control Theory, Birkhäuser Boston, Inc., 1995.

[14] H.T. Banks and R.C. Smith, "Implementation Issues Regarding PDE-Based Controllers - Control of Transient and Periodic Plate Vibrations," Center for Research in Scientific Computation Technical Report, CRSC-TR95-16, April 1995.

[15] T. Başar and P. Bernhard, $H^{\infty}$-Optimal Control and Related Minimax Design Problems, Birkhäuser, Boston, 1991.

[16] D.S. Bernstein and D.C. Hyland, The optimal projection equations for finite dimensional fixed order dynamic compensator of infinite dimensional systems, SIAM J. on Control and Optimization, 24, 1986, pp. 122-151.

[17] J. D'Cruz "The Active Control of Panel Vibrations with Piezoelectric Actuators," Proceedings of the Conference on Recent Advances in Adaptive and Sensory Materials and their Applications, VPI and SU, Blacksburg, VA, April 1992, pp. 665-674.

[18] C.R. Fuller, G.P. Gibbs and R.J. Silcox, "Simultaneous Active Control Flexural and Extension Waves in Beams," Journal of Intelligent Material Systems and Structures 1, April 1990, pp. 235-247.

[19] C.R. Fuller, C.H. Hansen and S.D. Snyder, "Active Control of Structurally Radiated Noise Using Piezoceramic Actuators," Proc. of Inter-Noise 89 (Newport Beach, CA), 1989, 509512.

[20] C.R. Fuller, S.D. Snyder, C.H. Hansen and R.J. Silcox, "Active Control of Interior Noise in Model Aircraft Fuselages Using Piezoceramic Actuators," AIAA 13 ${ }^{\text {th }}$ Aeroacoustics Conf. (Tallahassec) October 22-24, 1990, Paper 90-3922.

[21] W.L. Hallauer and S.E. Lamberson, "Experimental Active Vibration Damping of a Plane Truss Using Hybrid Actuation," Proc. 30th AIAA/ASME/ASCE/AHS/ASC: Structural Dynamics and Materials Conference, Paper 89-1169-CP, 1989, pP. 80-90.

[22] K. Ito, Finite-dimensional compensators for infinite-dimensional systems via Galerkintype approximation, SIAM J. on Control and Optimization, (28) 6, 1990, pp. 1251-1269. 
[23] B. van Keulen, $H_{\infty}$-Control for Distributed Parameter Systems: A State-Space Approach, Birkhäuser, Boston, 1993.

[24] S. Koshigoe and J.W. Murdock, "A Unified Analysis of both Active and Passive Damping for a Plate with Piezoelectric Transducers," Journal of the Acoustical Society of America, 93(1), 1993, pp. 346-355.

[25] H. Kwakernaak and R. Sivan, Linear Optimal Control System, John Wiley \& Sons, Inc., New York, 1972.

[26] J.D. Lambert, Numerical Methods for Ordinary Differential Systems: The Initial Value Problem, John Wiley \& Sons, Inc., New York, 1991.

[2T] E.H. Mansfield, The Bending and Stretching of Plates, Volume 6 in the International Series of Monographs on Aeronautics and Astronautics, The MacMillan Company, New York, 1964.

[28] V.L. Metcalf, U.S. Army Research Laboratory, NASA Langley Research Center, Personal Communication.

[29] R.C. Smith, "A Galerkin Method for Linear PDE Systems in Circular Geometries with Structural Acoustic Applications," ICASE Report No. 94-40, submitted to SIAM Journal on Scientific Computing. 


\begin{tabular}{|c|c|c|}
\hline \multicolumn{2}{|c|}{ REPORT DOCUMENTATION PAGE } & $\begin{array}{l}\text { Form Approved } \\
\text { OMB No. 0704-0188 }\end{array}$ \\
\hline \multicolumn{3}{|c|}{ 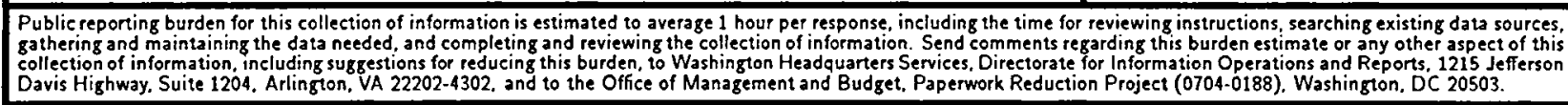 } \\
\hline 1. AGENCY USE ONLY(Leave blank) & \begin{tabular}{l|l|} 
2. REPORT DATE & 3. \\
May 1995 &
\end{tabular} & $\begin{array}{l}\text { JD DATES COVERED } \\
\text { jrt }\end{array}$ \\
\hline \multicolumn{2}{|c|}{$\begin{array}{l}\text { 4. TITLE AND SUBTITLE } \\
\text { EXPERIMENTAL CONFIRMATION OF A PDE-BASED } \\
\text { APPROACH TO DESIGN OF FEEDBACK CONTROLS }\end{array}$} & $\begin{array}{l}\text { 5. FUNDING NUMBERS } \\
\text { C NAS1-19480 } \\
\text { WU } 505-90-52-01\end{array}$ \\
\hline \multicolumn{2}{|c|}{$\begin{array}{l}\text { 6. AUTHOR(S) } \\
\text { H. T. Banks, Ralph C. Smith, D. E. Brown, R. J. Silcox, Vern L. Metcalf }\end{array}$} & \\
\hline \multicolumn{2}{|c|}{$\begin{array}{l}\text { 7. PERFORMING ORGANIZATION NAME(S) AND ADDRESS(ES) } \\
\text { Institute for Computer Applications in Science } \\
\text { and Engineering } \\
\text { Mail Stop 132C, NASA Langley Research Center } \\
\text { Hampton, VA 23681-0001 }\end{array}$} & $\begin{array}{l}\text { 8. PERFORMING ORGANIZATION } \\
\text { REPORT NUMBER } \\
\text { ICASE Report No. } 95-42\end{array}$ \\
\hline \multicolumn{2}{|c|}{$\begin{array}{l}\text { 9. SPONSORING/MONITORING AGENCY NAME(S) AND ADDRESS(ES) } \\
\text { National Aeronautics and Space Administration } \\
\text { Langley Research Center } \\
\text { Hampton, VA } 23681-0001\end{array}$} & $\begin{array}{l}\text { 10. SPONSORING/MONITORING } \\
\text { AGENCY REPORT NUMBER } \\
\text { NASA CR-198167 } \\
\text { ICASE Report No. } 95-42\end{array}$ \\
\hline
\end{tabular}

11. SUPPLEMENTARY NOTES

Langley Technical Monitor: Dennis M. Bushnell

Final Report

Submitted to SIAM Journal of Control and Optimization

12a. DISTRIBUTION/AVAILABILITY STATEMENT

12b. DISTRIBUTION CODE

Unclassified-Unlimited

Subject Category 64

13. ABSTRACT (Maximum 200 words)

Issues regarding the experimental implementation of PDE-based controllers are discussed in this work. While the motivating application involves the reduction of vibration levels for a circular plate through excitation of surfacemounted piezoceramic patches, the general techniques described here will extend to a variety of applications. The initial step is the development of a PDE model which accurately captures the physics of the underlying process. This model is then discretized to yield a vector-valued initial value problem. Optimal control theory is used to determine continuous-time voltages to the patches, and the approximations needed to facilitate discrete time implementation are addressed. Finally, experimental results demonstrating the control of both transient and steady state vibrations through these techniques are presented.

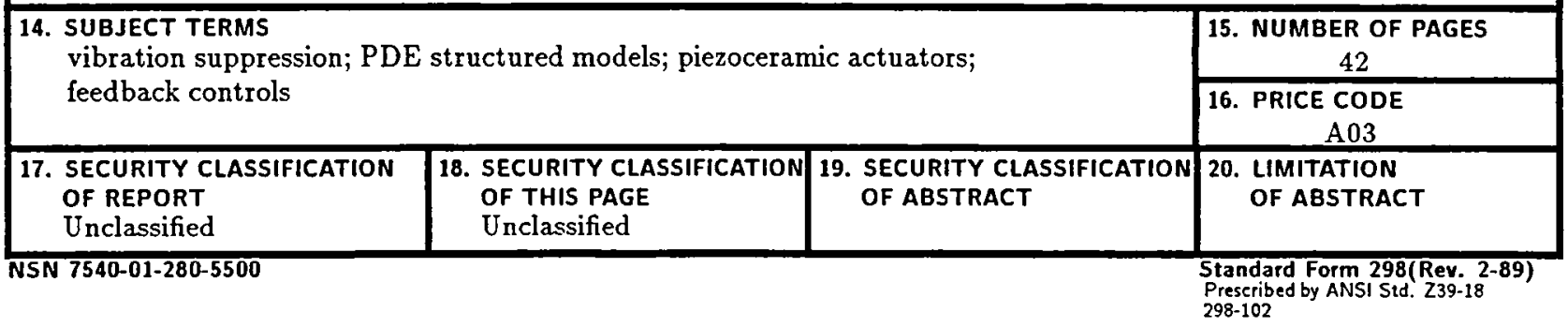


. 
